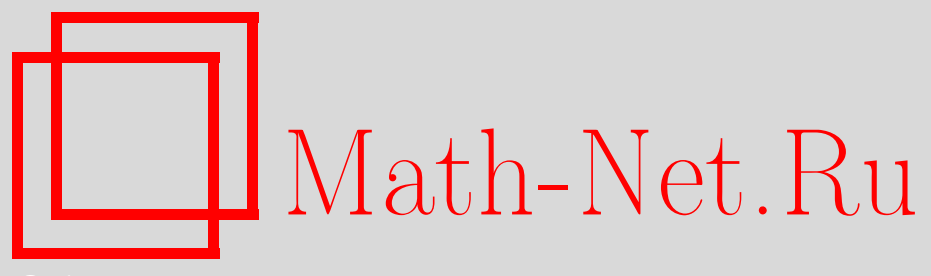

В. К. Захаров, А. В. Михалёв, Т. В. Родионов, Проблема Рисса-Радона-Фреше характеризации интегралов, УМН, 2010, том 65, выпуск 4, 153-178

DOI: https://doi.org/10.4213/rm9362

Использование Общероссийского математического портала Math-Net.Ru подразумевает, что вы прочитали и согласны с пользовательским соглашением http://www . mathnet.ru/rus/agreement

Параметры загрузки:

IP: 54.224 .135 .184

26 апреля 2023 г., 13:30:00

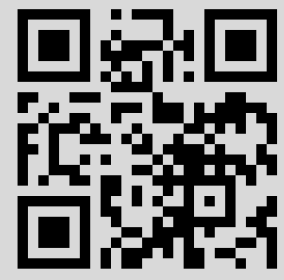




\title{
Проблема Рисса-Радона-Фреше характеризации интегралов
}

\author{
В. К. Захаров, А. В. Михалёв, Т. В. Родионов
}

Статья посвящена обзору результатов, связанных с задачей характеризации интегралов как линейных функционалов. Она восходит к известному результату Ф. Рисса (1909) об интегральном представлении ограниченных линейных функционалов интегралами Римана-Стилтьеса на отрезке и напрямую связана со знаменитой теоремой И. Радона (1913) об интегральном представлении ограниченных линейных функционалов интегралами Лебега на компакте в $\mathbb{R}^{n}$.

После работ И. Радона, М. Фреше и Ф. Хаусдорфа задача характеризации интегралов как линейных функционалов стала конкретизироваться как задача распространения теоремы Радона с $\mathbb{R}^{n}$ на более общие топологические пространства с радоновскими мерами. Эта задача оказалась трудной, и ее решение имеет долгую и богатую историю. Поэтому ее естественно называть проблемой Рисса-Радона-Фреше характеризации интегралов.

Важные этапы ее решения связаны с именами С. Банаха (1937-1938), С. Сакса (1937-1938), С. Какутани (1941), П. Халмоша (1950), Э. Хьюитта (1952), Р. Эдвардса (1953), Ю. В. Прохорова (1956), Н. Бурбаки (1969) и др. Существенные идейные и технические средства были разработаны А. Д. Александровым (1940-1943), М. Стоуном (1948-1949), Д. Фремлином (1974) и др.

Большая часть статьи посвящена современному этапу решения проблемы, связанному с работами Х. Кёнига (1995-2008), В.К. Захарова и А. В. Михалёва (1997-2009) и др. Общее решение проблемы изложено в виде параметрической теоремы о характеризации интегралов, из которой непосредственно следуют характеризационные теоремы указанных авторов.

Библиография: 60 названий.

Ключевые слова: радоновская мера, регулярная мера, радоновский интеграл, симметризуемые функции, узкий функционал, бимеры.

Работа выполнена при поддержке РФФИ (гранты № 08-01-00693, 08-01-00669) и программы "Ведущие научные школы" (грант НШ-3252.2010.1).

(C) В. К. ЗАхаров, А. В. Михалёв, Т. В. Родионов, 2010 


\section{СОДЕРЖАНИЕ}

1. Начальный этап характеризации интегралов как линейных функцио-

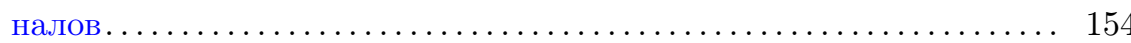

2. Этап ограниченных мер на компактных пространствах . . . . . . . 155

3. Этап неограниченных положительных мер на локально компактных пространствах .............................. 158

4. Этап ограниченных мер на некомпактных пространствах. Узкие функционалы . . . . . . . . . . . . . . . . . . . . . . . . . . . . . . 160

5. Переход к неограниченным положительным мерам на произвольных хаусдорфовых пространствах ..........................

6. Этап неограниченных положительных мер и ограниченных произвольных мер на хаусдорфовых пространствах .............. 165

7. Параметрическая характеризация интегралов. $\sigma$-точные функционалы 167

8. Этап произвольных мер на хаусдорфовом пространстве ........... 169

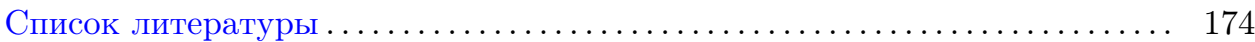

\section{1. Начальный этап характеризации интегралов как линейных функционалов}

Интерес к характеризации интегралов как линейных функционалов возник вместе с появлением интегралов Римана-Стилтьеса (1894).

Напомним, что действительнозначная функция $v:[a, b] \rightarrow \mathbb{R}$ на отрезке $[a, b]$ называется функцией ограниченной вариации, если

$$
\sup \left\{\sum\left(\left|v\left(x_{i+1}\right)-v\left(x_{i}\right)\right| \mid i=0, \ldots, n-1\right) \mid a \equiv x_{0}<x_{1}<\cdots<x_{n} \equiv b\right\}<\infty .
$$

Известно, что любая функция $v$ ограниченной вариации ограничена и представляется в виде разности $v_{1}-v_{2}$ двух ограниченных положительных возрастающих функций. Через $B V[a, b]$ обозначим линейное пространство всех функций ограниченной вариации на отрезке $[a, b]$.

Пусть $v$ - функция ограниченной вариации на $[a, b]$. Тогда интеграл Puмaна-Cmилтвеса $i_{v}(f) \equiv \int_{[a, b]} f d v$ дает линейный функционал $i_{v}$ на линейном пространстве $R I([a, b], v)$ всех функций $f:[a, b] \rightarrow \mathbb{R}$, интегрируемых по Риману-Стилтьесу относительно функции $v$.

Ясно, что $i_{v}(f)=i_{v_{1}}(f)-i_{v_{2}}(f)$, т. е. каждый интегральный функционал представляется в виде разности двух положительных интегральных функционалов.

Поскольку интегралов Римана-Стилтьеса $i_{v}$, соответствующих разным функциям $v$ ограниченной вариации, много и все они заданы на разных семействах функций $R I([a, b], v)$, естественно рассмотреть их на каком-либо одном хорошем линейном подпространстве $A[a, b]$ линейного пространства $\bigcap\{R I([a, b], v) \mid v \in$ $B V[a, b]\}$ всех универсально интегрируемых функций. Такое хорошее линейное подпространство имеется - это пространство $C[a, b]$ всех непрерывных на $[a, b]$ функций. 
Поэтому возникает семейство $I(C[a, b], B V[a, b]) \equiv\left\{i_{v}|C[a, b]| v \in B V[a, b]\right\}$ всех интегралов Римана-Стилтьеса на $C[a, b]$. Данное семейство является собственным линейным подпространством линейного пространства $(C[a, b])^{\times}$всех линейных функционалов на $C[a, b]$, т. е. $I(C[a, b], B V[a, b]) \varsubsetneqq(C[a, b])^{\times}$.

Поскольку эти интегралы являются чрезвычайно важными для математического анализа, Ж. Адамар (1903) [1] и М. Фреше (1904) [2] поставили и решали следующую естественную задачу (см. [3; Ист. очерк, с. 383]):

как охарактеризовать интеграль Римана-Стилтьеса среди всех линейных функционалов на $C[a, b]$ ?

Легко проверить, что пространство $I(C[a, b], B V[a, b])$ интегральных функционалов является подпространством линейного пространства $(C[a, b])^{\sim}$ всех ограниченных линейных функционалов на $C[a, b]$. Напомним, что функционал $\varphi: C[a, b] \rightarrow \mathbb{R}$ называется ограниченным, если $\sup \{|\varphi f| \mid f \in C[a, b]$ и $|f| \leqslant g\}<\infty$ для каждого $g \in C[a, b], g \geqslant 0$. Пытаясь доказать обратное вложение, Ж. Адамар в 1903 г. [1] показал, что каждый функционал $\varphi \in(C[a, b])^{\sim}$ представляется в виде $\varphi f=\lim _{n \rightarrow \infty} \int_{a}^{b} f(x) p_{n}(x) d x$, где $p_{n} \in C[a, b]$. Этот результат был уточнен М. Фреше [2].

Хорошо известное решение этой задачи было дано Ф. Риссом в 1909 г. [4] (см. также [5; гл. VIII, §8]).

Теорема 1. Пространство всех интегралов Римана-Стилтъеса на $C[a, b]$ совпадает с пространством всех линейных ограниченных функиионалов на $C[a, b]$, m.e. $I(C[a, b], B V[a, b])=(C[a, b])^{\sim} \varsubsetneqq(C[a, b])^{\times}$.

\section{2. Этап ограниченных мер на компактных пространствах}

С появлением интегралов Лебега-Радона задача характеризации интегралов как линейных функиионалов получила дальнейшее развитие.

В работе [6] И. Радон (1913), комбинируя идеи А. Лебега и Ф. Рисса, ввел вполне аддитивные функиии множества $\mu: \mathscr{M}_{\lambda^{n}}\left(\mathbb{R}^{n}\right) \rightarrow \mathbb{R}$, определенные на $\sigma$-алгебре всех множеств из $\mathbb{R}^{n}$, измеримых относительно меры Лебега $\lambda^{n}$. Обозначим семейство таких функций множеств через $\mathfrak{R F}\left(\mathbb{R}^{n}\right)$.

Для ограниченного множества $T$ из $\mathbb{R}^{n}$ Радон рассмотрел "вполне аддитивные функции множества" $\mu \mid T$, полученные сужением функций $\mu$ на $T$. Пользуясь конструкцией Лебега для $\mathbb{R}^{n}$, Радон определил интеграл $i_{\mu \mid T} f \equiv \int_{T} f(\mu \mid T)$, задающий линейный функционал на пространстве $C(T)$ всех непрерывных функций на $T$ (см. [3; Ист. очерк, с. 383]). Семейство $I(C(T), \mathfrak{R F}(T)) \equiv$ $\left\{i_{\mu \mid T}|C(T)| \mu \in \mathfrak{R F}\left(\mathbb{R}^{n}\right)\right\}$ всех интегралов Лебега-Радона на $C(T)$ является, как и ранее, собственным подпространством линейного пространства $C(T)^{\times}$.

В [6] И. Радон доказал следующую теорему.

Теорема 2. Пространство всех интегралов Лебега-Радона на $C(T)$ для замкнутого ограниченного (компактного) подпространства $T$ в $\mathbb{R}^{n}$ совпадает с пространством всех линейных ограниченных функционалов на $C(T)$, m.е. $I(C(T), \mathfrak{R F}(T))=C(T)^{\sim} \varsubsetneqq C(T)^{\times}$. 
"Почти сразу же после выхода в свет мемуара Радона Фреше замечает, что почти все результаты этой работы могут быть распространены на случай, когда «вполне аддитивная функция множества», вместо того чтобы быть определенной для измеримых подмножеств пространства $\mathbb{R}^{n}$, определена для некоторых подмножеств произвольного множества $E$ (эти подмножества таковы, что операции счетного объединения и разности снова дают множества, для которых функция определена)" [3; Ист. очерк, с. 383].

“... в 1915 г. Фреше определил в (IIа) (cм. [7] - авторы) «абстрактные» меры на множестве, с выделенной в нем $\sigma$-алгеброй подмножеств, и интегралы относительно этих мер..." [8; Ист. очерк, с. 581].

Упомянутое выше утверждение М. Фреше не касалось теоремы Радона, поскольку она относилась не к абстрактным, а к топологически регулярным мерам.

В 1914 г. вышла книга Ф. Хаусдорфа [9], в которой были заложены основы теории топологических пространств. И. Радон существенно использовал следующее топологическое свойство вполне аддитивной функции множества $\mu \mid T$ : для каждого $M \in \mathscr{M} \mid T$ и любого $\varepsilon>0$ найдутся замкнутое множество $F$ и открытое множество $G$ такие, что $F \subset M \subset G$ и $|\mu| T \mid(G \backslash F)<\varepsilon$. В дальнейшем это свойство было названо свойством регулярности, а $\sigma$-аддитивные функции множеств (т. е. меры) с этим свойством получили название радоновских (см. [10; п. 18.2.1] и [11; п. 7.1]).

После упомянутых работ И. Радона и М. Фреше задача характеризации интегралов как линейных функционалов стала конкретизироваться как задача распространения теоремы Радона с $\mathbb{R}^{n}$ на более общие топологические пространства с регулярными мерами.

Эта задача оказалась трудной, и ее решение имеет долгую и богатую историю. Поэтому ее естественно называть проблемой Рисса-Радона-Фреше характеризации интегралов. Далее в статье последовательно описываются различные этапы ее решения.

Первое существенное продвижение в решении этой проблемы было получено С. Банахом в 1937 г. Используя введенные М. Фреше вполне аддитивные функции множества (абстрактные меры) и топологическое свойство регулярности, С. Банах обобщил теорему Радона на случай компактного метрического пространства (приложение II к монографии [12]), тот же результат получен и С. Саксом [13] (см. [14; гл. IV, п. 16]). В 1941 г. С. Какутани [15] обобщил результат Банаха и Сакса на произвольное компактное пространство.

Для изложения этих результатов приведем необходимые математические понятия.

Пусть $T$ - множество, $\mathscr{M}-\sigma$-алгебра подмножеств $T$. Всякое счетноаддитивное отображение $\mu: \mathscr{M} \rightarrow[a, b] \subset \mathbb{R}$ называется (широкой) ограниченной мерой [11; п. 1.3.2]. Для ограниченной меры $\mu$ имеет место разложение Хана $T=T_{+} \cup T_{-}$такое, что $v_{+}(\mu) \equiv \mu\left(M \cap T_{+}\right) \geqslant 0$ и $v_{-}(\mu) \equiv \mu\left(M \cap T_{-}\right) \leqslant 0$ для любого $M \in \mathscr{M}\left[11 ;\right.$ п. 3.1.1]. Отображения $v_{+}(\mu), v_{-}(\mu)$ и $|\mu| \equiv v_{+}(\mu)-v_{-}(\mu)$ являются ограниченными мерами на $\mathscr{M}$ и называются положительной, отрищательной и полной вариациями меры $\mu$ соответственно. При этом спра- 
ведливо разложение Жордана $\mu=v_{+}(\mu)+v_{-}(\mu)$ (см. [11; п. 3.1] и [14; гл. III, п. 4.11]).

Обозначим через $\mathfrak{M}_{b}(T, \mathscr{M})$ множество всех ограниченных мер на $\sigma$-алгебре $\mathscr{M}$. Для ограниченных мер $\mu$ и $\nu$ определяются следующие операции:

1) $(\mu+\nu) M \equiv \mu M+\nu M$;

2) $(r \mu) M \equiv r \mu M$ для любого $r \in \mathbb{R}$;

3) $(\mu \vee \nu) M \equiv \sup \{\mu L+\nu N \mid L, N \in \mathscr{M}$ и $L \cup N=M$ и $L \cap N=\varnothing\}$;

4) $(\mu \wedge \nu) M \equiv \inf \{\mu L+\nu N \mid L, N \in \mathscr{M}$ и $L \cup N=M$ и $L \cap N=\varnothing\}$.

Относительно этих операций множество $\mathfrak{M}_{b}(T, \mathscr{M})$ является решеточным и линейным пространством. При этом справедливы равенства $v_{+}(\mu)=\mu_{+} \equiv$ $\mu \vee 0$ и $v_{-}(\mu)=\mu_{-} \equiv \mu \wedge \mathbf{0}$ (см. [16; пп. VIII.1.3 и VIII.2.3]).

Любые непустые семейства $\mathscr{E}$ подмножеств $E$ множества $T$ будем называть ансамблями ${ }^{1}$. Через $\mathscr{P}(T)$ обозначим ансамбль всех подмножеств $T$. Для произвольного ансамбля $\mathscr{E}$ на $T$ через $M(T, \mathscr{E})$ обозначим семейство всех $\mathscr{E}$-измеримых функций на $T$, т. е. таких, что $f^{-1}[] x, y[] \in \mathscr{E}$ для любого $] x, y[\subset \mathbb{R}$.

Для любой положительной функции $f: T \rightarrow \mathbb{R}_{+} \equiv[0, \infty[$ и любой положительной ограниченной меры $\mu: \mathscr{M} \rightarrow[a, b] \subset \mathbb{R}_{+}$число

$$
i_{\mu}(f) \equiv \int f d \mu \equiv \sup \left\{\sum\left(\inf \left(f\left[M_{i}\right]\right) \mu M_{i} \mid i \in I\right)\right\} \in[0, \infty]
$$

называется интегралом Лебега(-Радона-Фреше) функиии $f$ относительно ограниченной меры $\mu$, где супремум берется по всем конечным разбиениям $\left(M_{i} \in \mathscr{M} \mid i \in I\right)$ множества $T$ (см. [17; п. 12.2]).

Решеточное линейное пространство всех $\mathscr{M}$-измеримых функций $f$ таких, что $\int f_{+} d \mu<\infty$ и $\int\left(-f_{-}\right) d \mu<\infty$, где $f_{+} \equiv f \vee \mathbf{0}$ и $f_{-} \equiv f \wedge \mathbf{0}$, обозначим через $M I(T, \mathscr{M}, \mu)$. Для произвольной функции $f \in M I(T, \mathscr{M}, \mu)$ число $i_{\mu}(f) \equiv \int f d \mu \equiv \int f_{+} d \mu-\int\left(-f_{-}\right) d \mu$ называется интегралом от $f$ относительно $\mu$. Для произвольной ограниченной меры $\mu$ и любой функции $f \in M I(T, \mathscr{M}, \mu) \equiv M I\left(T, \mathscr{M}, v_{+}(\mu)\right) \cap M I\left(T, \mathscr{M},-v_{-}(\mu)\right)$ интегралом от $f$ относительно $\mu$ называется число $i_{\mu}(f) \equiv \int f d \mu \equiv \int f d\left(v_{+}(\mu)\right)-\int f d\left(-v_{-}(\mu)\right)$.

Пусть $(T, \mathscr{G})$ - компактное топологическое пространство с ансамблями $\mathscr{G}, \mathscr{F}$, $\mathscr{C}$ и $\mathscr{B}$ открытых, замкнутых, компактных и борелевских подмножеств соответственно. Пусть $\mathscr{M}-\sigma$-алгебра, содержащая $\mathscr{G}$. Ограниченная мера $\mu$ на $\mathscr{M}$ была названа радоновской, если для каждого $M \in \mathscr{M}$ и любого $\varepsilon>0$ найдутся $F \in \mathscr{F}$ и $G \in \mathscr{G}$ такие, что $F \subset M \subset G$ и $|\mu|(G \backslash F)<\varepsilon[10 ;$ п. 18.2.1].

Множество всех ограниченных радоновских мер $\mu: \mathscr{M} \rightarrow[a, b]$ на топологическом пространстве $(T, \mathscr{G})$ обозначим через $\mathfrak{R M}_{b}(T, \mathscr{G}, \mathscr{M})$. Наряду с $\mathfrak{R M}_{b}(T, \mathscr{G}, \mathscr{B})$ иногда будем писать кратко $\mathfrak{R} \mathfrak{M}_{b}(T, \mathscr{G})$. Непосредственно проверяется, что семейство $\mathfrak{R M}_{b}(T, \mathscr{G}, \mathscr{M})$ является решеточным линейным подпространством пространства $\mathfrak{M}_{b}(T, \mathscr{M})$.

\footnotetext{
${ }^{1}$ Мы используем термин "ансамбль" ("еnsemble", в переводе с французского, - "множество") для семейств множеств и оставляем слово “семейство” для семейств функций, функционалов и мер.
} 
Интегралы $i_{\mu}$ относительно ограниченных радоновских мер будем называть (ограниченными) радоновскими интегралами. Так как интерес представляют различные семейства радоновских интегралов для различных подмножеств $R M(T, \mathscr{G})$ множества $\mathfrak{R M}_{b}(T, \mathscr{G})$, то естественно рассмотреть пространство $U I(T, \mathscr{G}, R M(T, \mathscr{G})) \equiv \cap(M I(T, \mathscr{B}, \mu) \mid \mu \in R M(T, \mathscr{G}))$ всех универсально интегрируемых функицй относительно множества $R M(T, \mathscr{G})$. Тогда для любого линейного подпространства $A(T) \subset U I(T, \mathscr{G}, R M(T, \mathscr{G}))$ каждая радоновская мера $\mu \in R M(T, \mathscr{G})$ задает линейный функционал $i_{\mu} \mid A(T): A(T) \rightarrow \mathbb{R}$.

Линейное подпространство $A(T)$ пространства $U I(T, \mathscr{G}, R M(T, \mathscr{G}))$ назовем разделяющим меры из множества $R M(T, \mathscr{G})$, если отображение $\mu \mapsto i_{\mu} \mid A(T)$ из $R M(T, \mathscr{G})$ в $A(T)^{\times}$является инъективным.

Для множества $R M(T, \mathscr{G})$ и разделяющего его меры линейного пространства $A(T) \subset U I(T, \mathscr{G}, R M(T, \mathscr{G}))$ можно рассмотреть семейство $I(A(T), R M(T, \mathscr{G})) \equiv$ $\left\{i_{\mu}|A(T)| \mu \in R M(T, \mathscr{G})\right\}$ всех радоновских интегралов на пространстве $A(T)$ относительно множества $R M(T, \mathscr{G})$.

Теперь мы можем дать точную формулировку результата Банаха-Сакса-Какутани ([15], см. также [10; п. 18.4.1] и [14; гл. IV, п. 6.3]).

Теорема 3. Для любого компактного топологического пространства $(T, \mathscr{G})$ пространство всех ограниченных радоновских интегралов на $C(T, \mathscr{G})$ совпадает с пространством всех ограниченных линейных функционалов на $C(T, \mathscr{G})$, m.e. $I\left(C(T, \mathscr{G}), \mathfrak{R M}_{b}(T, \mathscr{G})\right)=C(T, \mathscr{G})^{\sim} \varsubsetneqq C(T, \mathscr{G})^{\times}$.

Более того, отображение $\mu \mapsto i_{\mu} \mid C(T, \mathscr{G})$ является изоморфизмом решеточных линейных пространств $\mathfrak{R M}_{b}(T, \mathscr{G})$ и $C(T, \mathscr{G})^{\sim}$.

Данная теорема закрыла этап ограниченных радоновских мер на компактных пространствах.

Заметим, что в теоремах Рисса, Радона и Банаха-Сакса-Какутани наиболее трудной частью является интегральное представление функиионала (точнее, восстановление меры), т. е. построение функции $v$ и меры $\mu$ по функционалу $\varphi$ так, что $\varphi=i_{v} \mid C[a, b]$ и $\varphi=i_{\mu} \mid C(T, \mathscr{G})$.

\section{3. Этап неограниченных положительных мер на локально компактных пространствах}

K сожалению, теорема Какутани не затрагивала самый знаменитый интегральный функционал $i_{\lambda}(f) \equiv \int_{\mathbb{R}} f d \lambda$ по лебеговой мере $\lambda$ на всей вещественной оси $\mathbb{R}$. Пространство $\mathbb{R}$ не является компактным, и мера Лебега $\lambda: \mathscr{B}(\mathbb{R}) \rightarrow \overline{\mathbb{R}}_{+} \equiv[0, \infty]$ не является ограниченной.

Свойства меры Лебега $\lambda$ стали отправным пунктом при определении положительной не обязательно ограниченной радоновской меры $\mu: \mathscr{B}(T, \mathscr{G}) \rightarrow \overline{\mathbb{R}}_{+}$ на топологическом пространстве $(T, \mathscr{G})$. Положительная мера $\mu: \mathscr{B} \rightarrow \overline{\mathbb{R}}_{+}$была названа радоновской, если она конечна на компактных подмножествах $T$ и обладает свойством компактной регулярности, т. е. $\mu B=\sup \{\mu C \mid C \in \mathscr{C} \wedge C \subset B\}$ для любого $B \in \mathscr{B} \equiv \mathscr{B}(T, \mathscr{G})$ (см. [18; п. 73A], [19; пп. 411В и 411H(b)], [8; гл. Х, §3, п. 2]). 
В отличие от $\mathfrak{R M} \mathfrak{M}_{b}(T, \mathscr{G})$, семейство $\mathfrak{R M}(T, \mathscr{G})_{0}$ всех положительных радоновских мер на $(T, \mathscr{G})$ не является линейным пространством, а является только конусом, т. е. для любых $\mu_{1}, \mu_{2} \in \mathfrak{R M}(T, \mathscr{G})_{0}$ и любых $r_{1}, r_{2} \in \mathbb{R}_{+}$справедливо $r_{1} \mu_{1}+r_{2} \mu_{2} \in \mathfrak{R M}(T, \mathscr{G})_{0}$.

В этом случае радоновский интеграл определяется простой формулой

$$
i_{\mu}(f) \equiv \int f d \mu \equiv \int f_{+} d \mu-\int\left(-f_{-}\right) d \mu
$$

для любых борелевских функций $f$ и мер $\mu \in \mathfrak{R M}(T, \mathscr{G})_{0}$, где интеграл от положительной функции по положительной мере определяется аналогично разделу 2.

Использование только положительных мер влечет за собой необходимость рассматривать интегралы не только как ограниченные, но и как положительные функционалы. Если $X(T) \subset F(T)$, то функционал $\varphi: X(T) \rightarrow \mathbb{R}$ называется положительным, если из $f \in X(T)$ и $f \geqslant 0$ следует $\varphi f \geqslant 0$. Ясно, что любой положительный функционал является ограниченным. Более того, по теореме Рисса-Канторовича любой ограниченный функционал $\varphi$ является разностью $\varphi_{+}-\left(-\varphi_{-}\right)$двух положительных функционалов $\varphi_{+} \equiv \varphi \vee 0$ и $-\varphi_{-} \equiv(-\varphi) \vee \mathbf{0}$, где $\varphi_{-} \equiv \varphi \wedge \mathbf{0}-$ отрицательный функционал (см. [10; п. 3.6.5] и [20; п. VIII.2.1]).

В 1950-53 гг. П. Халмош [21], Э. Хьюитт [22] и Р. Эдвардс [23] получили следующий результат.

Теорема 4. Пусть (Т, $\mathscr{G})$ - локально компактное пространство. Тогдаконус всех интегралов $i_{\mu}$ по положительным (не обязательно ограниченным) радоновским мерам $\mu$ на $(T, \mathscr{G})$ совпадает с конусом всех положительных линейных функиионалов на пространстве $A(T)=C_{c}(T, \mathscr{G})$ всех непрерывных функций на $T$ с компактными носителями, m.е. $I\left(C_{c}(T, \mathscr{G}), \mathfrak{R M}(T, \mathscr{G})_{0}\right)=$ $\left(C_{c}(T, \mathscr{G})^{\sim}\right)_{+}$.

Более того, отображение $\mu \mapsto i_{\mu} \mid C_{c}(T, \mathscr{G})$ является изоморфизмом решеточных конусов $\mathfrak{R M}(T, \mathscr{G})_{0} u\left(C(T, \mathscr{G})^{\sim}\right)_{+}$.

Эта теорема в некоторых изданиях стала называться теоремой Рисса о представлении [17; п. 12.36].

Отметим, что доказательство этой теоремы ничем принципиальным не отличалось от доказательства теоремы Какутани. Именно по этой причине авторы ограничились случаем локально компактного пространства и положительных мер на нем, а их доказательства были непригодными для более общих пространств и более общих мер.

Несущественность продвижения на локально компактные пространства и положительные неограниченные меры породила естественный интерес к обобщению теоремы Рисса-Радона-Банаха-Сакса-Какутани-Халмоша-Хьюитта-Эдвардса на: 1) более широкий класс топологических пространств и 2) более широкий класс "радоновских" мер.

Дальнейший существенный прорыв в решении проблемы был сделан для случая ограниченных радоновских мер на тихоновских пространствах. 


\section{4. Этап ограниченных мер на некомпактных пространствах. Узкие функционалы}

Если $(T, \mathscr{G})$ - тихоновское (вполне регулярное) пространство, то пространство $C_{c}(T, \mathscr{G})$ нельзя использовать, так как возможно равенство $C_{c}(T, \mathscr{G})=\{\mathbf{0}\}$ и поэтому $C_{c}(T, \mathscr{G})$ не будет разделять радоновские меры (т. е. всем радоновским мерам будет соответствовать один тривиальный нулевой функционал). В связи с этим можно взять за основу пространство $C_{b}(T, \mathscr{G})$ всех ограниченных непрерывных функций. Но для такого пространства имеет смысл рассматривать только линейное пространство $I\left(C_{b}(T, \mathscr{G}), \mathfrak{R M}_{b}(T, \mathscr{G})\right)$ интегралов по всем ограниченным радоновским мерам.

В этом случае равенство Рисса-Радона уже несправедливо, поскольку

$$
I\left(C_{b}(T, \mathscr{G}), \mathfrak{R M}_{b}(T, \mathscr{G})\right) \varsubsetneqq C_{b}(T, \mathscr{G})^{\sim} .
$$

Поэтому нужно искать новое характеристическое свойство радоновских интегралов.

В 1969 г. Н. Бурбаки (см. [8; гл. IX, § 5, п. 2]), опираясь на идеи Ю. В. Прохорова [24], возникшие при изучении слабой компактности семейств ограниченных радоновских мер (см. [11; п. 8.6]), выделили такое новое свойство.

Будем использовать обозначение $F(T)$ для семейства всех функций $f: T \rightarrow \mathbb{R}$. Пусть $A(T) \subset F(T)$ - некоторое функциональное решеточное линейное пространство на $T$. Через $A(T)_{+}, A_{b}(T)$ и $A_{c}(T)$ будем обозначать его подпространства, состоящие из неотрицательных функций, ограниченных функций и функций с компактными носителями соответственно.

Функционал $\varphi$ на $A(T)$ называется узким (tight) [18; 73G(e)] или обладающим свойством Прохорова [25], если для любого $\varepsilon>0$ существует компактное подмножество $C \subset T$ такое, что условия $f \in A(T)$ и $|f| \leqslant \chi(T \backslash C)$ влекут $|\varphi f|<\varepsilon$. Множество всех ограниченных узких линейных функционалов на $A(T)$ обозначим через $A(T)^{\pi}$. Н. Бурбаки доказали следующую теорему [8; гл. IX, §5, п. 2].

ТЕОРема 5. Пространство всех радоновских интегралов по ограниченным радоновским мерам на тихоновском пространстве $(T, \mathscr{G})$ совпадает с пространством всех узких ограниченных линейных функиионалов на пространстве $C_{b}(T, \mathscr{G})$ всех ограниченных непрерывных функций на T, m.е. $I\left(C_{b}(T, \mathscr{G})\right.$, $\left.\mathfrak{R M}_{b}(T, \mathscr{G})\right)=C_{b}(T, \mathscr{G})^{\pi}$.

Более того, отображение $\mu \mapsto i_{\mu} \mid C_{b}(T, \mathscr{G})$ является изоморбизмом решеточных линейных пространств $\mathfrak{R M}_{b}(T, \mathscr{G})$ u $C_{b}(T, \mathscr{G})^{\pi}$.

В книге Д. Фремлина эта теорема названа теоремой Прохорова $[18 ; 73 \mathrm{G}(\mathrm{e})]$.

Отметим, что для компактного пространства $(T, \mathscr{G})$ справедливо равенство $C_{b}(T, \mathscr{G})^{\pi}=C_{b}(T, \mathscr{G})^{\sim}$. Поэтому теорема Н. Бурбаки является естественным обобщением теоремы С. Какутани.

Эта теорема полностью закрыла линию Рисса-Радона-Банаха-Сакса-Какутани по описанию ограниченных радоновских интегралов в виде функционалов на пространстве $C_{b}(T, \mathscr{G})$. 


\section{5. Переход к неограниченным положительным мерам на произвольных хаусдорфовых пространствах}

Несмотря на существенное продвижение в случае ограниченных радоновских интегралов на тихоновском пространстве, характеризация неограниченных интегралов на тихоновском пространстве, ограниченных интегралов на произвольном хаусдорфовом пространстве и, тем более, неограниченных интегралов на произвольном хаусдорфовом пространстве оставалось неизвестной.

Во-первых, было неясно, какие неограниченные "радоновские" меры надо рассматривать. Во-вторых, не было ясно, на каком линейном пространстве функций $A(T)$ следует рассматривать все "радоновские" интегралы $i_{\mu}$. Если пространство $(T, \mathscr{G})$ - не тихоновское, то пространство $C_{b}(T, \mathscr{G})$ не подходит, поскольку оно может состоять только из постоянных функций, и, соответственно, не разделять радоновские меры. Если же $(T, \mathscr{G})$ - тихоновское, но меры неограниченные, то $C_{b}(T, \mathscr{G})$ не подходит, поскольку $i_{\mu}(\mathbf{1})=\infty$, а пространство $C_{c}(T, \mathscr{G})$ не подходит, поскольку оно может состоять только из нулевой функции.

Таким образом, нужно было найти, во-первых, подходящее определение общей радоновской меры, во-вторых, подходящее пространство не обязательно непрерывных функций $A(T)$, заменяющее отыгравшее свою роль пространство непрерывных функций $C(T, \mathscr{G})$, и, в-третьих, новое характеристическое свойство радоновских интегралов $i_{\mu}$ на этом пространстве.

В 1970 г. Ф. Топсо [26] и в 1974 г. Д. Фремлин [18; 72Е] продвинулись в вопросе об интегральном представлении линейных поточечно $\sigma$-непрерывных положительных функционалов на абстрактных функциональных пространствах $A(T)$. Опишем их результаты.

Функционал $\varphi$ на $A(T)$ называется поточечно $\sigma$-непрерывным, если для каждой функции $f \in A(T)$ и каждой монотонной последовательности $\left(f_{m} \in\right.$ $\left.A(T) \mid m \in M \subset \mathbb{N}_{0}\right)$ условие $\left(f_{m}(t) \mid m \in M\right) \rightarrow f(t)$ для каждого $t \in T$ влечет $\left(\varphi f_{m} \mid m \in M\right) \rightarrow \varphi f$ (см. [16; гл. I, п. 8.1]). В [11; п. 7.9.2] линейные функционалы на $C_{b}(T, \mathscr{G})$ с такими свойствами называются $\sigma$-гладкими. Все поточечно $\sigma$-непрерывные функционалы ограничены.

Пусть $T$ - множество, $\mathscr{E}-$ некоторый ансамбль на $T$. Всякое отображение (функцию множеств) $\varepsilon: \mathscr{E} \rightarrow \overline{\mathbb{R}} \equiv[-\infty, \infty]$ будем называть оцениванием на $\mathscr{E}$. Оценивание $\varepsilon$ назовем

- положительным, если $\operatorname{rng} \varepsilon \equiv\{\varepsilon E \mid E \in \mathscr{E}\} \subset \overline{\mathbb{R}}_{+} \equiv[0, \infty]$,

- конечным, если $\operatorname{rng} \varepsilon \subset \mathbb{R}$,

- ограниченным, если $\operatorname{rng} \varepsilon \subset[a, b] \subset \mathbb{R}$.

Оценивание $\varepsilon: \mathscr{E} \rightarrow \overline{\mathbb{R}}$ назовем натуралъным, если $\varnothing \in \mathscr{E}, \varepsilon(\varnothing)=0$ и rng $\varepsilon$ лежит либо в $[-\infty, \infty[$, либо в $]-\infty, \infty]$.

Натуральное $\sigma$-аддитивное оценивание, определенное на некотором кольце, $\delta$-кольце или $\sigma$-алгебре, будем называть мерой, узкой мерой или широкой мерой соответственно. 
Пусть $\mathscr{R}$ - мультипликативный и $\sigma$-аддитивный ансамбль на $T$. Обозначим через $\mathfrak{L}(T, \mathscr{R})$ семейство всех оцениваний $\lambda: \mathscr{R} \rightarrow \mathbb{R}_{+}$, обладающих следующими свойствами:

1) $\lambda(E \cup D) \geqslant \lambda E+\lambda D$ для любых $E, D \in \mathscr{R}$ таких, что $E \cup D \in \mathscr{R}$ и $E \cap D=\varnothing$;

2) $\lambda(\varnothing)=0$ и $\left(\lambda E_{n} \mid n \in \mathbb{N}_{0}\right) \downarrow 0$ для каждой последовательности $\left(E_{n} \in \mathscr{R} \mid\right.$ $\left.n \in \mathbb{N}_{0}\right) \downarrow \varnothing ;$

3) $\lambda H \leqslant \lambda L \leqslant \lambda H+\sup \{\lambda R \mid R \in \mathscr{R}$ и $R \subset L \backslash H\}$, если $H, L \in \mathscr{R}$ и $H \subset L$.

Определим оценивание $\nu: \mathscr{P}(T) \rightarrow \overline{\mathbb{R}}_{+}$, полагая $\nu E \equiv \sup \{\lambda R \mid R \in \mathscr{R}$ и $R \subset E\}$. Рассмотрим ансамбль $\mathscr{M} \equiv\{M \in \mathscr{P}(T) \mid \forall L \in \mathscr{R}(\lambda L \leqslant \nu(L \cap M)+$ $\nu(L \backslash M))\}$ и оценивание $\mu \equiv e \lambda \equiv \nu \mid \mathscr{M}$.

$\mathrm{C}$ каждым оцениванием $\varepsilon: \mathscr{E} \rightarrow \overline{\mathbb{R}}$ свяжем два подансамбля:

$$
\mathscr{E}^{0}(\varepsilon) \equiv\{E \in \mathscr{E} \mid \varepsilon E=0\} \quad \text { и } \quad \mathscr{E}^{f}(\varepsilon) \equiv\{E \in \mathscr{E} \mid \varepsilon E \in \mathbb{R}\} .
$$

Обозначим $\mathscr{N}_{0}(T, \mathscr{E}, \varepsilon) \equiv\left\{N_{0} \in \mathscr{P}(T) \mid \forall E \in \mathscr{E}\left(N_{0} \cap E \in \mathscr{E}^{0}(\varepsilon)\right)\right\}$. Будем называть $\varepsilon$ полным, если $\left\{N \in \mathscr{P}(T) \mid \exists N_{0} \in \mathscr{N}_{0}\left(N \subset N_{0}\right)\right\} \subset \mathscr{E}$ (см. [27; п. 5.2]).

Пусть $\mathscr{R} \subset \mathscr{E}$. Ансамбль $\mathscr{E}$ называется $\mathscr{R}$-насыщенным, если $\{U \in \mathscr{P}(T) \mid$ $\forall R \in \mathscr{R}(U \cap R \in \mathscr{E})\} \subset \mathscr{E}$. Оценивание $\varepsilon: \mathscr{E} \rightarrow \overline{\mathbb{R}}$ называется

(а) $\mathscr{R}$ - $\sigma$-непрерывным сверху, если $\left(\varepsilon R_{n} \mid n \in \mathbb{N}_{0}\right) \downarrow \varepsilon R$ для каждого множества $R \in \mathscr{R}$ и любой последовательности $\left(R_{n} \in \mathscr{R} \mid n \in \mathbb{N}_{0}\right) \downarrow R$;

(b) внутренне $\mathscr{R}$-регулярным, если $\varepsilon E=\sup \{\varepsilon R \mid(R \in \mathscr{R} \cap \mathscr{E} f(\varepsilon))$ и $(R \subset E)\}$ для всех $E \in \mathscr{E}$ (см. [18; п. 73G(i)], [16; п. V.1.1]);

(c) $\mathscr{R}$-насыщенным, если $\mathscr{E}$ является $\mathscr{R}$-насыщенным (см. [18; п. 71А(B)], [27; п. 1.6]).

$\mathscr{E}^{f}(\varepsilon)$-насыщенное оценивание назовем силъно насыщенным (оно называется насыщенным в [11; п. 1.12.121] и локально определенным в [18; п. 64G(c)]).

Как было сказано в разделе 2 , восстановление меры по функционалу является трудной задачей ("hard analysis"). Следующий результат (теорема Фремлина о продолжении оцениваний до мер) является ключевым для решения этой задачи [18; п. 71A].

ТЕОРема 6. Пусть $\mathscr{R}$ - мультипликативный и б-аддитивный ансамбль на T. Тогда для каждого оченивания $\lambda \in \mathfrak{L}(T, \mathscr{R})$ ансамбль $\mathscr{M}$ является $\sigma$-алгеброй, а $\mu \equiv е \lambda: \mathscr{M} \rightarrow \overline{\mathbb{R}}_{+}-$полной, сильно насыщенной, внутренне $\mathscr{R}$-регулярной и $\mathscr{R}$-насыщенной мерой, продолжающей $\lambda$.

Пусть $A(T)$ - линейное пространство функций на $T$ и $\varphi$ - линейный функционал на $A(T)$. Пусть $\mathscr{M}-\sigma$-алгебра на $T$ и $\mu-$ (широкая) мера на $\mathscr{M}$. Функционал $\varphi$ называется представимым интегралом Лебега над измеримым пространством $(T, \mathscr{M}, \mu)$ или интегрально представимым относительно меры $\mu$, если $A(T) \subset M I(T, \mathscr{M}, \mu)$ и $\varphi f=i_{\mu} f$ для всех $f \in A(T)$.

Функциональное семейство $A(T)$ называется усекаемым (или обладающим свойством Стоуна), если условие $f \in A(T)$ влечет $f \wedge \mathbf{1} \in A(T)$ (см. [18; п. 71D] и [16; п. I.7.2]). Например, пространство $C(T, \mathscr{G})=M(T, \mathscr{G})$ всех непрерывных функций и его подпространства $C_{b}(T, \mathscr{G}), C_{c}(T, \mathscr{G})$ являются таковыми. 
Будем говорить, что семейство $A(T)$ огибает $[\sigma$-огибает $]$ сверху функцию $h \in F(T)$, если существует сеть $\left(f_{m} \in A(T) \mid m \in M\right)$ [последовательность $\left.\left(f_{m} \in A(T) \mid m \in M \subset \omega\right)\right]$ такая, что $\left(f_{m}(t) \mid m \in M\right) \downarrow h(t)$ для любого $t \in T$. Соответственно, будем говорить, что $A(T)$ огибает $[\sigma$-огибает] снизу функцию $g \in F(T)$, если $\left(f_{m}(t) \mid m \in M\right) \uparrow g(t)$ для любого $t \in T$.

Рассмотрим ансамбль $\mathscr{I}^{\sigma}(A(T))$ всех множеств $R \subset T$ таких, что семейство $A(T) \quad \sigma$-огибает сверху функцию $\chi(R)$. Для любого положительного функционала $\varphi$ на $A(T)$ можно определить на $\mathscr{I}^{\sigma}(A(T))$ положительное конечное оценивание $\lambda \equiv \Lambda \varphi$, полагая $\lambda R \equiv \inf \{\varphi f \mid f \in A(T)$ и $f \geqslant \chi(R)\}$ для любого $R \in \mathscr{I}^{\sigma}(A(T))$.

Следующий важный результат, опирающийся на теорему Фремлина, может быть назван теоремой Александрова-Стоуна об интегральном представлении, так как он восходит к работам [28] и [29]. М. Стоун показал важность поточечной $\sigma$-непрерывности функционала для существования меры, а А. Д. Александров показал важность внутренней регулярности меры для ее единственности.

Теорема 7. Пусть $A(T)$ - усекаемое решеточное линейное пространство функций на Т и ч- положителъный поточечно б-непрерывный линейный функиионал на $A(T)$. Тогда

1) $\lambda \in \mathfrak{L}(T, \mathscr{R})$, где $\mathscr{R} \equiv \mathscr{I}^{\sigma}(A(T))$;

2) мера $\mu \equiv е \lambda: \mathscr{M} \rightarrow \overline{\mathbb{R}}_{+}$является полной, сильно насыщенной, внутренне $\mathscr{R}$-регулярной, $\mathscr{R}$-насыщенной и $\mathscr{R}$ - $\sigma$-непрерывной сверху;

3) функиионал ч представим интегралом Лебега над $(T, \mathscr{M}, \mu)$;

4) мера н единственна в следующем смысле: если существует другая полная, сильно насыщенная, внутренне $\mathscr{R}$-регулярная мера $\nu: \mathscr{N} \rightarrow \overline{\mathbb{R}}_{+}$такая, что ч представим интегралом Лебега над $(T, \mathscr{N}, \nu)$, mо $\mathscr{N}=\mathscr{M} u \nu=\mu$.

Доказательство этой теоремы можно найти в [18; пп. $71 \mathrm{G}$ и $72 \mathrm{~F}]$.

Таким образом, для всякого усекаемого решеточного линейного пространства $A(T)$ определено отображение $R \equiv e \circ \Lambda: \varphi \mapsto \mu$, ставящее в соответствие каждому положительному поточечно $\sigma$-непрерывному функционалу на $A(T)$ меру с указанными выше свойствами.

Несмотря на свою силу и красоту, эта теорема не позволяет описывать семейства радоновских интегралов $I(A(T), R M(T, \mathscr{G}))$, поскольку:

1) построенная мера $\mu=R \varphi$ не обязана быть радоновской;

2) если даже она радоновская, произвольное усекаемое решеточное линейное пространство $A(T)$ вовсе не гарантирует разделения радоновских мер в смысле раздела 2;

3) по радоновскому интегральному функционалу вида $\varphi \equiv i_{\varepsilon}$ не обязательно восстанавливается исходная мера $\varepsilon$.

В [26], [30] Д. Поллард и Ф. Топсо применили результаты типа теоремы 7 при изучении радоновского представления на произвольном хаусдорфовом пространстве, ограничившись конусом положительных непрерывных функций. Однако, на таком пространстве непрерывных функций может быть слишком мало. Зато на нем имеется достаточное число полунепрерывных функций. 
Достаточное в том смысле, что они разделяют одновременно и точки пространства, и радоновские меры.

Семейство всех полунепрерывных сверху функииц $f: T \rightarrow \mathbb{R}$, т. е. таких, что $f^{-1}[]-\infty, x[] \in \mathscr{G}$ для каждого $x \in \mathbb{R}$, обозначим через $S C^{u}(T, \mathscr{G}) . \mathrm{K}$ сожалению, это семейство не является линейным пространством, а является лишь конусом, т. е. его элементы можно только складывать и умножать на положительные числа.

Использование конуса $S C^{u}(T, \mathscr{G})_{+}$всех положительных полунепрерывных сверху функций позволило Ф. Топсо [31], [32], а также Б. Анже и К. Портенье [33] получить некоторые достаточные условия для представления функционалов радоновскими интегралами на произвольном хаусдорфовом пространстве.

Однако, использование функциональных конусов $K(T)$ вместо линейных пространств $A(T)$ влечет за собой необходимость рассматривать интегралы не как линейные, а только как конусно-линейные функционалы. Если $K(T)-$ конус, то функционал $\varphi: K(T) \rightarrow \mathbb{R}$ называется конусно-линейным, если для любых $f, f_{1}, f_{2} \in K(T)$ и $r_{1}, r_{2} \in \mathbb{R}_{+}$из равенства $f=r_{1} f_{1}+r_{2} f_{2}$ следует $\varphi f=r_{1} \varphi f_{1}+r_{2} \varphi f_{2}$.

На этом пути Х. Кёниг [34]-[36] получил в 1995-2000 гг. конусную версию теоремы Рисса-Радона о представлении функционалов интегралами.

Скажем, что конусно-линейный положительный функционал $\varphi: K(T) \equiv$ $S C_{c}^{u}(T, \mathscr{G})_{+} \rightarrow \mathbb{R}$ обладает свойством Кёнига, если выполнены два условия:

1) для всех $f, g \in K(T)$ таких, что $f \leqslant g$, верно неравенство

$$
\begin{aligned}
\varphi g-\varphi f \leqslant \sup \{\inf \{ & \left.\varphi u_{m} \mid m \in M\right\} \mid\left(u_{m} \in A(T) \mid m \in M\right) \downarrow \\
& \wedge \forall m \in M\left(u_{m} \leqslant f\right) \\
& \left.\wedge \forall t \in T\left(\inf \left\{u_{m} \mid m \in M\right\} \leqslant g(t)-f(t)\right)\right\} ;
\end{aligned}
$$

$2) \inf \left\{\varphi(f \wedge x \mathbf{1}) \mid x \in \mathbb{R}_{+}\right\}=0$ для всех $f \in K(T)$.

Семейство всех таких функционалов обозначим через $\left(S C_{c}^{u}(T, \mathscr{G})_{+}\right)^{\varkappa}$.

ТЕОРема 8 [36; теорема 3.7]. Для любого хаусдорфова пространства $(T, \mathscr{G})$ справедливо равенство $I\left(S C_{c}^{u}(T, \mathscr{G})_{+}, \mathfrak{R M}(T, \mathscr{G})_{0}\right)=\left(S C_{c}^{u}(T, \mathscr{G})_{+}\right)^{\varkappa}$.

В последующих работах Кёнига [37], [38] весьма сложное условие 1) практически не изменилось.

Теорема Кёнига не дала решения проблемы Рисса-Радона-Фреше описания радоновских интегралов даже для положительных мер. Причиной этого является отсутствие у конусного семейства $K(T)$ важнейшего свойства линейности, присутствовавшего в описаниях Рисса, Радона, Банаха, Сакса, Какутани, Халмоша, Хьюитта, Эдвардса, Бурбаки и др. 


\section{6. Этап неограниченных положительных мер и ограниченных произвольных мер на хаусдорфовых пространствах}

Подходящее для решения проблемы Рисса-Радона-Фреше линейное функциональное пространство $A(T)$ было введено В. К. Захаровым в 1989-1990 гг. в статьях [39], [40], посвященных задаче Хаусдорфа-Серпинского.

Поскольку семейство $S C^{u}(T, \mathscr{G})$ является лишь конусом, еще Ф. Хаусдорф в 1915 г. решал задачу погружения конуса $S C^{u}(T, \mathscr{G})$ всех полунепрерывных сверху функций в линейное пространство. Он рассмотрел семейство $S C(T, \mathscr{G}) \equiv$ $S C^{u}(T, \mathscr{G})-S C^{u}(T, \mathscr{G}) \equiv\left\{f-g \mid f, g \in S C^{u}(T, \mathscr{G})\right\}$ и доказал, что оно является решеточным линейным пространством [41]. Далее В. Серпинский в 1921 г. установил, что пространство $S C(T, \mathscr{G})$ не является полным относительно равномерной сходимости. Поэтому он рассмотрел равномерное пополнение $\overline{S C(T, \mathscr{G})}$ и дал его описание через суммы абсолютно сходящихся рядов из $C(T, \mathscr{G})$, не дав никакого функционального описания (см. [42] и [9; п. 37.5]).

Задача функционального описания пространства $\overline{S C(T, \mathscr{G})}$ (задача Xaycдорфа-Серпинского) остается открытой до сих пор. Поскольку пространство $\overline{S C(T, \mathscr{G})}$ является избыточным для задач теории меры, В. К. Захаровым [39] было введено более узкое решеточное линейное пространство $S C^{b}(T, \mathscr{G}) \equiv$ $\left\{f-g \mid f, g \in S C_{b}^{u}(T, \mathscr{G})\right\}$ ограниченнъх полунепрерывных функиий и рассмотрено его равномерное пополнение $\overline{S C^{b}(T, \mathscr{G})}$ относительно равномерной нормы $\|\cdot\|_{u}$. Задачу функционального описания пространства $\overline{S C^{b}(T, \mathscr{G})}$ естественно назвать ограниченной задачей Хаусдорфа-Серпинского. Она была решена в работе [39].

Пусть $(T, \mathscr{G})$ - топологическое пространство. Рассмотрим мультипликативный ансамбль $\mathscr{K} \equiv\{G \cap F \mid G \in \mathscr{G}$ и $F \in \mathscr{F}\}$ всех симметризуемых множеств $K \equiv G \cap F$ (см. также [43], [44]). С ансамблем $\mathscr{K}$ свяжем булеву алгебру $\mathscr{A}$ всех александровских множеств, т. е. таких, которые являются конечными объединениями симметризуемых множеств. Эта алгебра широко использовалась А. Д. Александровым в работе [28].

Колебанием функции $f$ на множестве $S$ будем называть величину $\omega(f, S) \equiv$ $\sup \{|f(s)-f(t)| \mid s, t \in S\}$. Функцию $f: T \rightarrow \mathbb{R}$ назовем симметризуемой, если для любого $\varepsilon>0$ существует конечное покрытие $\left(K_{i} \in \mathscr{K} \mid i \in I\right)$ множества $T$ такое, что $\omega\left(f, K_{i}\right)<\varepsilon$ для любого $i \in I$ [39]. Симметризуемые функции являются частным случаем равномерных функиий или, иначе говоря, функиий малого колебания (о них см., например, [45]-[49]).

Пространство $S(T, \mathscr{G})$ всех симметризуемых функций на $(T, \mathscr{G})$ является линейным, решеточным и усекаемым [39]. Кроме того, справедливы равенство $S_{b}(T, \mathscr{G})=S(T, \mathscr{G})$ и вложения $C_{b}(T, \mathscr{G}) \equiv M_{b}(T, \mathscr{G}) \subset M_{b}(T, \mathscr{K}) \subset S(T, \mathscr{G}) \subset$ $M_{b}\left(T, \mathscr{K}_{\sigma}\right)[49]$.

Именно пространство $S(T, \mathscr{G})$ дает решение ограниченной задачи Хаусдорфа-Серпинского.

TEOPEMA 9 [39]. $\overline{S C^{b}(T, \mathscr{G})}=S(T, \mathscr{G})$.

Это равенство объясняет, почему функции из $S(T, \mathscr{G})$ были вначале названы метаполунепрерьвными [50], [51]. 
Отметим, что если $(T, \mathscr{G})$ - произвольное хаусдорфово пространство, то возможна тривиализация $C_{c}(T, \mathscr{G})=\{\mathbf{0}\}$. В то же время пространство $S_{c}(T, \mathscr{G})$ нетривиально и разделяет и точки, и меры.

Действительно, характеристические функции $\chi\left(\left\{t_{0}\right\}\right)$ одноточечных множеств $\left\{t_{0}\right\}$ являются полунепрерывными сверху и, следовательно, лежат в пространстве $S_{c}(T, \mathscr{G})$. Ясно, что они разделяют точки из $T$.

Что касается разделения мер, то для любых различных радоновских мер $\mu_{1}$ и $\mu_{2}$ существует борелевское множество $B$ такое, что $\mu_{1} B \neq \mu_{2} B$. В силу компактной регулярности найдется компактное множество $C \subset B$ такое, что $\mu_{1} C \neq \mu_{2} C$. Ясно, что $f \equiv \chi(C) \in S_{c}(T, \mathscr{G})$ и $i_{\mu_{1}} f=\mu_{1} C \neq \mu_{2} C=i_{\mu_{2}} f$. Следовательно, $i_{\mu_{1}}\left|S_{c}(T, \mathscr{G}) \neq i_{\mu_{2}}\right| S_{c}(T, \mathscr{G})$.

Это означает, что класс усекаемых решеточных линейных пространств $A(T) \subset S(T, \mathscr{G})$ является подходящим (в смысле раздела 5) для решения общей проблемы Рисса-Радона-Фреше.

После обретения подходящего класса $A(T) \subset S(T, \mathscr{G})$ осталась задача выделения характеристических свойств интегралов $i_{\mu} \mid A(T)$ (см. начало раздела 5).

С этой целью в 1997 г. В. К. Захаров и А. В. Михалёв в [51] ввели понятие тонкого функционала (см. также [47]). Линейный функционал $\varphi$ на решеточном линейном пространстве $A(T)$, содержащем $S_{c}(T, \mathscr{G})$, называется внутренне компактным, если для каждого $\varepsilon>0$ и каждой убывающей последовательности множеств $\left(A_{n} \in \mathscr{A} \mid n \in \mathbb{N}\right)$ такой, что $\left\{\chi\left(A_{n} \backslash C\right) \mid n \in \mathbb{N}, C \in \mathscr{C}\right\} \subset A(T)$, найдутся $n_{0} \in \mathbb{N}$ и $C \in \mathscr{C}$ такие, что $C \subset \bigcap\left(A_{n} \mid n \in \mathbb{N}\right)$ и $\varphi \chi\left(A_{n_{0}} \backslash C\right)<\varepsilon$. Поточечно $\sigma$-непрерывный и внутренне компактный функционал называется тонким. Семейство всех тонких функционалов на $A(T)$ обозначим через $A(T)^{\zeta}$.

Появление этих новых семейств функций и функционалов позволило получить для произвольного хаусдорфова пространства следующие характеризации радоновских интегралов [51]-[54], [47].

Теорема 10. Пусть $(T, \mathscr{G})$ - хаусдорфово пространство. Тогда

1) $I\left(S_{c}(T, \mathscr{G}), \mathfrak{R M}(T, \mathscr{G})_{0}\right)=\left(S_{c}(T, \mathscr{G})^{\zeta}\right)_{+}$;

2) $I\left(S_{c}(T, \mathscr{G}), \mathfrak{R M}_{b}(T, \mathscr{G})\right)=S_{c}(T, \mathscr{G})^{\complement}$;

3) $I\left(S(T, \mathscr{G}), \mathfrak{R M}_{b}(T, \mathscr{G})\right)=S(T, \mathscr{G})^{\complement}=S(T, \mathscr{G})^{\zeta}$.

Более того, возникающие здесъ соответствия между мерами и функиионалами являются изоморфизмами соответствующих решеточных конусов или пространств.

Здесь через $A(T)^{(5)}$ обозначено подсемейство равномерно ограниченных тонких функционалов на $A(T)$. Функционал $\varphi$ мы называем равномерно ограниченным на $A(T)$, если он ограничен и $\sup \{|\varphi f| \mid f \in A(T)$ и $|f| \leqslant \mathbf{1}\}<\infty$. Если решеточное линейное пространство $A(T)$ содержит $\mathbf{1}$, то всякий ограниченный функционал на нем будет равномерно ограниченным.

Теорема 10 дала решение проблемы Рисса-Радона-Фреше для положительных мер на произвольном хаусдорфовом пространстве. 
В [53], [55], [56] из приведенного описания были выведены как следствия описания Рисса-Радона-Какутани, Халмоша-Хьюитта-Эдвардса и Бурбаки, что продемонстрировало силу и общность теоремы 10.

\section{7. Параметрическая характеризация интегралов. $\sigma$-точные функционалы}

Описания, данные в теоремах 2-5 с использованием пространства $C(T, \mathscr{G})$ непрерывных функций, и описания, данные в теореме 10 с использованием пространства $S(T, \mathscr{G})$ симметризуемых функций, являются различными и по форме и по доказательству. В связи с этим возникла потребность в получении объединяющей параметрической теоремы с функциональным пространством $A(T) \subset S(T, \mathscr{G})$ в качестве параметра, из которой все вышеприведенные результаты о линейном радоновском представлении получались бы как непосредственные следствия (частные случаи).

Поскольку пространства $C(T, \mathscr{G})$ непрерывных функций и $S(T, \mathscr{G})$ симметризуемых функций весьма различны, было необходимо выделить некоторые абстрактные свойства параметра $A(T) \subset U I(T, \mathscr{G}, \cdot)$, существенные для характеризации семейств $I\left(A(T), \mathfrak{R} \mathfrak{M}_{b}(T, \mathscr{G})\right)$ и $I\left(A(T), \mathfrak{R M}(T, \mathscr{G})_{0}\right)$. Эти свойства огибания $(E)$ и $\left(E_{\sigma}\right)$ и Дини $(D)$ были выделены в работах [25], [57].

Семейство $A(T)$ имеет свойство $(E)$ [соответственно $\left.\left(E_{\sigma}\right)\right]$, если выполнены следующие три условия:

(i) для любых $G \in \mathscr{G}$ и $u \in A(T)+$ семейство $A(T)$ огибает [ $\sigma$-огибает] снизу функцию $\chi(G) \wedge u$;

(ii) для любых $F \in \mathscr{F}, C \in \mathscr{C}$ и $u \in A(T)_{+}$семейство $A(T)$ огибает [ $\sigma$-огибает] сверху функции $\chi(F) \wedge u$ и $\chi(C)$;

(iii) для любого $G \in \mathscr{G}$ и любого компактного подмножества $C \subset G$ найдется такая функция $v \in A(T)$, что $\chi(C) \leqslant v \leqslant \chi(G)$.

Ясно, что свойство $\left(E_{\sigma}\right)$ является более сильным, чем свойство $(E)$.

Все конкретные функциональные пространства, использованные в теоремах 1-5 и 10, обладают абстрактными свойствами $(E)$ или $\left(E_{\sigma}\right)$.

ПРЕДЛОЖЕНИЕ 1 [57]. Справедливы следующие утверждения:

1) если $(T, \mathscr{G})$ - локально компактное пространство, то $C_{c}(T, \mathscr{G})$ обладает свойством $(E)$;

2) если $(T, \mathscr{G})$ - тихоновское пространство, то $C_{b}(T, \mathscr{G})$ обладает свойством $(E)$;

3) если $(T, \mathscr{G})$ - хаусдорбово пространство, то $S(T, \mathscr{G})$ и $S_{c}(T, \mathscr{G})$ обладают свойством $\left(E_{\sigma}\right)$.

Семейство $A(T)$ обладает свойством Дини $(D)$, если из поточечной сходимости сети $\left(f_{m} \in A(T) \mid m \in M\right)$ к функции $f \in A(T)$, вытекает, что эта сходимость равномерна на каждом компактном множестве $C \in \mathscr{C}$.

Функционал $\varphi$ на $A(T)$ называется локально узким (или обладающим локальным свойством Прохорова), если для любых $G \in \mathscr{G}, u \in A(T)_{+}$и $\varepsilon>0$ 
существует компактное множество $C \subset G$ такое, что условия $f \in A(T)$ и $|f| \leqslant$ $\chi(G \backslash C) \wedge u$ влекут $|\varphi f|<\varepsilon$.

Функционал $\varphi$ на $A(T)$ называется $\sigma$-точным, если он является поточечно $\sigma$-непрерывным и локально узким [25], [57]. Множество всех $\sigma$-точных линейных функционалов на $A(T)$ будем обозначать через $A(T)^{\triangle}$, а его подмножество равномерно ограниченных функционалов через $\left.A(T)^{(}\right)$.

Радоновский интеграл $i_{\mu}$, рассматриваемый как функционал на семействе $S I(T, \mathscr{G}, \mu)$ всех симметризуемых функиий, интегрируемых относителъно $\mu$, обладает свойством $\sigma$-точности.

ПреДЛОЖЕНИЕ 2 [57]. Пусть $(T, \mathscr{G})$ - хаусдорфово пространство. Тогда:

1) если $\mu \in \mathfrak{R M}(T, \mathscr{G})_{0}$, mо $C_{c}(T, \mathscr{G}) \subset S_{c}(T, \mathscr{G}) \subset S I(T, \mathscr{G}, \mu)$ и интегральный функиионал $i_{\mu} \mid S_{c}(T, \mathscr{G})$ является $\sigma$-точным;

2) если $\mu \in \mathfrak{R M}_{b}(T, \mathscr{G})$, то $C_{b}(T, \mathscr{G}) \subset S(T, \mathscr{G})=S I(T, \mathscr{G}, \mu)$ и интегралъный функиионал $i_{\mu} \mid S(T, \mathscr{G})$ является $\sigma$-точным.

Отметим, что новое свойство $\sigma$-точности функционалов фактически содержится в более простых свойствах функционалов быть ограниченными и узкими, использованных ранее при решении задачи Рисса-Радона-Фреше в классических случаях (см. теоремы 4 и 5 ).

ПредлоЖениЕ 3 [57], [58]. Справедливы следующие утверждения:

1) если $(T, \mathscr{G})$ - локально компактное пространство, то

$$
C_{c}(T, \mathscr{G})^{\sim}=C_{c}(T, \mathscr{G})^{\Delta}
$$

2) если $(T, \mathscr{G})$ - тихоновское пространство, то

$$
\left.C_{b}(T, \mathscr{G})^{\pi}=C_{b}(T, \mathscr{G})^{\Delta}=C_{b}(T, \mathscr{G})^{(}\right)
$$

3) если $(T, \mathscr{G})$ - хаусдорфово пространство, то

$$
S(T, \mathscr{G})^{\zeta}=S(T, \mathscr{G})^{\Delta}=S(T, \mathscr{G})^{\otimes} \quad u \quad S_{c}(T, \mathscr{G})^{\zeta}=S_{c}(T, \mathscr{G})^{\Delta} .
$$

Свойства $(E) \&(D)$ и $\left(E_{\sigma}\right)$ функционального пространства $A(T) \subset S(T, \mathscr{G})$ и свойство $\sigma$-точности радоновского интеграла $i_{\mu} \mid A(T)$ позволили доказать следующую параметрическую теорему с параметром $A(T)$ [25], [57].

Теорема 11. Пусть $(T, \mathscr{G})$ - хаусдорфово пространство и $A(T)$-усекаемое решеточное линейное подпространство в $S(T, \mathscr{G})$, обладающее свойством $\left(E_{\sigma}\right)$ или свойством $(E) \&(D)$. Тогда:

1) $I\left(A(T), \mathfrak{R M}(T, \mathscr{G})_{0}\right)=\left(A(T)^{\Delta}\right)_{+}$для $A(T) \subset U I\left(T, \mathscr{G}, \mathfrak{R M}(T, \mathscr{G})_{0}\right)$;

2) $I\left(A(T), \mathfrak{R M}_{b}(T, \mathscr{G})\right)=A(T)^{\oplus}$.

Более того, возникающие здесь соответствия между мерами и функиионалами являются изоморфизмами соответствующих решеточных конусов или пространств.

Из этой параметрической теоремы и предложений 1, 2 и 3 вытекают уже как частные случаи теоремы 2-5 и 10 о характеризации радоновских интегралов. Более того, эта теорема при $A(T)=S_{c}(T, \mathscr{G})$ и $A(T)=S(T, \mathscr{G})$ дает 
новые описания радоновских интегралов как для положительных, так и для произвольных ограниченных радоновских мер на хаусдорфовом пространстве, благодаря использованию $\sigma$-точных функционалов.

Доказательство теоремы 11 проводится по следующей схеме.

Рассмотрим усекаемое решеточное линейное подпространство

$$
B(T) \equiv S_{\mathrm{m}}(T, \mathscr{G}, A(T)) \equiv\{f \in S(T, \mathscr{G}) \mid \exists u \in A(T)(|f| \leqslant u)\} \subset S(T, \mathscr{G}) .
$$

Используя упрощенный вариант метода Даниэля продолжения функционала, можно доказать следующую теорему [57].

TеОрема 12. Пусть $(T, \mathscr{G})$ - хаусдорфово пространство и $A(T)$ - усекаемое решеточное линейное подпространство в $S(T, \mathscr{G})$, обладающее свойством $\left(E_{\sigma}\right)$ или свойством $(E) \&(D)$. Тогда:

1) для каждого б-точного линейного функционала $\varphi$ на $A(T)$ существует единственный $\sigma$-точный линейный функционал $\varphi_{S}$ на $B(T)$, расширяющий функиионал $\varphi$;

2) отображение $Q: \varphi \mapsto \varphi_{S}$ является изоморфизмом между решеточными линейными пространствами $A(T)^{\triangle}$ и $B(T)^{\Delta}$.

Если $\varphi$ - произвольный $\sigma$-точный линейный функционал, то для положительных функционалов $\varphi_{1} \equiv \varphi \vee \mathbf{0}$ и $\varphi_{2} \equiv(-\varphi) \vee \mathbf{0}$ можно рассмотреть соответствующие положительные меры $\mu_{1}(\varphi) \equiv R \varphi_{1}$ и $\mu_{2}(\varphi) \equiv R \varphi_{2}$ из теоремы 7 , примененной к усекаемому решеточному линейному пространству $B(T)$. Рассмотрим общую радоновскую меру $\mu \equiv \mu_{1}(\varphi)-\mu_{2}(\varphi)$. Для нее справедлива следующая теорема.

Теорема 13. Пусть $(T, \mathscr{G})$ - хаусдорфово пространство и $A(T)$ - усекаемое решеточное линейное подпространство в $S(T, \mathscr{G})$, обладающее свойством $\left(E_{\sigma}\right)$ или свойством $(E) \&(D)$. Тогда функционал $\varphi_{S}: S_{\mathrm{m}}(T, \mathscr{G}, A(T)) \rightarrow \mathbb{R}$ является интегрально представимым относительно меры $\mu$.

СледСтвиЕ. Пусть $(T, \mathscr{G})$ - хаусдорфово пространство и $A(T)$ - усекаемое решеточное линейное подпространство в $S(T, \mathscr{G})$, обладающее свойством $\left(E_{\sigma}\right)$ или свойством $(E) \&(D)$. Тогда:

1) положительный функиионал $\varphi: A(T) \rightarrow \mathbb{R}$ является интегрально представимым относительно положительной меры $\mu_{1}(\varphi)$;

2) равномерно ограниченный функиионал $\varphi: A(T) \rightarrow \mathbb{R}$ является интегрально представимым относительно ограниченной меры $\mu=\mu_{1}(\varphi)-\mu_{2}(\varphi)$.

\section{8. Этап произвольных мер на хаусдорфовом пространстве}

Все рассмотренные выше радоновские меры были либо ограниченными, либо положительными. Понятие произвольной радоновской меры со значениями в $\overline{\mathbb{R}}$ отсутствовало в литературе; оно появилось в 2002 г. в работе В. К. Захарова [25].

Пусть $T$ - множество и $\mathscr{M}-\sigma$-алгебра на $T$. Согласно разделу 5 , оценивание $\mu: \mathscr{M} \rightarrow \overline{\mathbb{R}}$ называется (широкой) мерой, если $\mu(\varnothing)=0, \operatorname{rng} \mu$ лежит либо в $[-\infty, \infty[$, либо в $]-\infty, \infty]$ и $\mu$ является $\sigma$-аддитивным [14; п. III.4.3]. 
В книге [14; пп. III.4.10 и III.4.11] показано, что для таких мер сохраняется разложение Хана, понятие вариаций и разложение Жордана $\mu=v_{+}(\mu)+v_{-}(\mu)$ из раздела 2 ; при этом по крайней мере одна из вариаций меры $\mu$ конечна.

Отсюда следует, что для конечной меръ $\mu: \mathscr{M} \rightarrow \mathbb{R}$ обе вариации конечны и, значит, ограничены. Следовательно, сама мера $\mu$ тоже ограничена. Это означает, что любая конечная мера является ограниченной. Именно поэтому кажущийся более широким случай конечных мер на самом деле совпадает со случаем ограниченных мер.

Для конечной меры $\mu$ определена ее полная вариация $|\mu| \equiv v_{+}(\mu)+v_{-}(\mu)$ и справедливы равенства $\mu_{+} \equiv \mu \vee \mathbf{0}=v_{+}(\mu)$ и $\mu_{-} \equiv \mu \wedge \mathbf{0}=v_{-}(\mu)[16$; п. VIII.2.3].

Пусть $(T, \mathscr{G})$ - хаусдорфово пространство. В соответствии с [25], [57], мы называем меру $\mu: \mathscr{M} \rightarrow \overline{\mathbb{R}}$ (широкой) радоновской мерой на $(T, \mathscr{G})$, если выполнены следующие условия:

1) $\mathscr{G} \subset \mathscr{M}$

2) $\mathscr{C} \subset \mathscr{M}^{f}(\mu)$, т. е. $\mu C \in \mathbb{R}$ для любого $C \in \mathscr{C}$;

3) для любого $M \in \mathscr{M}$ такого, что $\mu M \in \mathbb{R}$, и любого $\varepsilon>0$ существует $C \in \mathscr{C}$ такое, что $C \subset M$ и $|\mu M-\mu C|<\varepsilon$;

4) для любого $M \in \mathscr{M}$ такого, что $\mu M=\infty$ [соответственно $\mu M=-\infty$ ], и любого $a \in \mathbb{R}$ существует $C \in \mathscr{C}$ такое, что $C \subset M$ и $\mu C>a$ [соответственно $\mu C<a]$.

В силу первого условия $\sigma$-алгебра $\mathscr{M}$ содержит борелевскую $\sigma$-алгебру $\mathscr{B}$. Для случая положительных мер совокупное свойство 3) \& 4) равносильно свойству компактной регулярности, которое ранее использовалось при определении положительных радоновских мер (см. раздел 3 ).

Такое общее понятие произвольной радоновской меры позволяет вернуться к классической линии Рисса-Радона-Банаха-Сакса-Какутани, поскольку для компактного пространства оно равносильно понятию ограниченной радоновской меры из раздела 2. Это видно из следующих утверждений.

ПредЛОЖЕНИЕ 4 [58]. Пусть $(T, \mathscr{G})$ - хаусдорфово пространство, $\mathscr{M}$ $\sigma$-алгебра, содержащая $\mathscr{G}$, и $\mu$-ограниченная мера на $\mathscr{M}$. Тогда следующие свойства равносильны:

1) $\mu$ - радоновская мера в определенном выше смысле;

2) для каждого $M \in \mathscr{M}$ и любого $\varepsilon>0$ найдутся $C \in \mathscr{C} u G \in \mathscr{G}$ такие, что $C \subset M \subset G u|\mu|(G \backslash C)<\varepsilon$.

СЛЕДСТВИЕ. Пусть $(T, \mathscr{G})$ - компактное пространство, $\mathscr{M}-\sigma$-алгебра, содержащая $\mathscr{G}, и \mu$-ограниченная мера на $\mathscr{M}$. Тогда следующие утверждения равносильны:

1) $\mu$ - радоновская мера в определенном выше смысле;

2) $\mu$ - ограниченная радоновская мера в смысле раздела 2.

Семейство всех радоновских мер на $(T, \mathscr{G})$ обозначим через $\mathfrak{R M}(T, \mathscr{G})$. K сожалению, оно не является линейным пространством, поскольку на $\overline{\mathbb{R}}$ нет операции $\infty-\infty$. 
Общий радоновский интеграл $i_{\mu} \equiv i_{v_{+}(\mu)}-i_{-v_{-}(\mu)}$ по произвольной радоновской мере $\mu$, рассматриваемый как функционал на семействе $S I(T, \mathscr{G}, \mu)$, обладает, как и ранее в разделе 6 , свойством $\sigma$-точности. Поэтому для функционального параметра $A(T)$ из параметрической теоремы 11 справедливо вложение

$$
I(A(T), \mathfrak{R M}(T, \mathscr{G})) \subset A(T)^{\triangle} .
$$

Поскольку семейство радоновских мер $\mathfrak{R M}(T, \mathscr{G})$ не является линейным пространством, соответствующее семейство радоновских интегралов $I(A(T)$, $\mathfrak{R M}(T, \mathscr{G}))$ в силу условия разделения мер из раздела 2 тоже не является линейным пространством. Значит, это семейство $I(A(T), \mathfrak{R M}(T, \mathscr{G}))$ не может исчерпывать все линейное пространство $\sigma$-точных функционалов $A(T)^{\Delta}$.

Следовательно, нужно найти еще уточняющее характеристическое свойство (которое пока будем обозначать (?)) общих радоновских интегралов $i_{\mu}$, выделяющее семейство $I(A(T), \mathfrak{R M}(T, \mathscr{G}))$ из всего линейного пространства $A(T)^{\Delta}$ как некоторое подсемейство $\left(A(T)^{\Delta}\right)(?)$.

Тогда восстановление общей радоновской меры $\mu$ по соответствующему функционалу $\varphi \in\left(A(T)^{\Delta}\right)_{(?)}$ естественно проводить следующим образом. По положительным функционалам $\varphi_{+}$и $-\varphi_{-}$можно восстановить положительные радоновские меры $\mu_{1}(\varphi)$ и $\mu_{2}(\varphi)$ из утверждения 1) теоремы 11 и взять радоновскую меру $\mu=\mu_{1}(\varphi)-\mu_{2}(\varphi)$. Но для этого нужно знать, что в паpe $\left(\mu_{1}(\varphi), \mu_{2}(\varphi)\right)$ положительных радоновских мер одна из мер конечна. Пока это не установлено, приходится рассматривать пару $\left(\mu_{1}(\varphi), \mu_{2}(\varphi)\right)$ как самостоятельный объект. Точнее говоря, произвольному $\sigma$-точному функционалу $\psi \in A(T)^{\triangle}$ естественно сопоставить не радоновскую меру $\mu=\mu_{1}(\psi)-\mu_{2}(\psi)$, а некоторую "бимеру" $\mathfrak{M} \leadsto\left(\mu_{1}(\psi), \mu_{2}(\psi)\right)$.

Таким образом, для решения задачи описания семейства $I(A(T), \mathfrak{R M}(T, \mathscr{G}))$ необходимо сначала разобраться с семейством всех радоновских "бимер" на произвольном хаусдорфовом пространстве $(T, \mathscr{G})$. Это было сделано В. К. Захаровым и А. В. Михалёвым в 1997-1999 гг. в [47], [51].

Напомним, что подмножество $P$ из $T$ называется предкомпактным (относительно компактным), если $\mathrm{cl} P$ является компактным, т. е. $P$ содержится в некотором компактном множестве. Подансамбль ансамбля $\mathscr{B}(T, \mathscr{G})$, состоящий из всех предкомпактных борелевских множеств, обозначим через $\mathscr{B}_{c} \equiv$ $\mathscr{B}_{c}(T, \mathscr{G})$. Он является $\delta$-кольцом.

Конечная узкая мера $\mu: \mathscr{B}_{c} \rightarrow \mathbb{R}$ называется узкой радоновской мерой на $(T, \mathscr{G})$ [51; п. II.1], если для любого $M \in \mathscr{B}_{c}$ и любого $\varepsilon>0$ существует $C \in \mathscr{C}$ такое, что $C \subset M$ и $|\mu M-\mu C|<\varepsilon$. Подробнее об узких радоновских мерах можно прочитать в [47], [51].

Триплет $\beta \equiv\left(\mu, \mu_{1}, \mu_{2}\right)$, состоящий из мер $\mu, \mu_{1}$ и $\mu_{2}$ на $(T, \mathscr{G})$, называется радоновским триплетом на хаусдорфовом пространстве $(T, \mathscr{G})$, если:

1) $\mu_{1}: \mathscr{B} \rightarrow \overline{\mathbb{R}}_{+}$и $\mu_{2}: \mathscr{B} \rightarrow \overline{\mathbb{R}}_{+}$являются положительными широкими радоновскими мерами;

2) $\mu: \mathscr{B}_{c} \rightarrow \overline{\mathbb{R}}$ является узкой радоновской мерой;

3) $\mu M=\mu_{1} M-\mu_{2} M$ для каждого $M \in \mathscr{B}_{c}$.

Если $\mu_{1}$ и $\mu_{2}$ ограничены, то триплет $\beta$ назовем ограниченным. 
Множество всех радоновских триплетов на $(T, \mathscr{G})$ обозначим через $\mathfrak{R}(T, \mathscr{G})$. Подсемейство ограниченных радоновских триплетов обозначим через $\mathfrak{R}_{b}(T, \mathscr{G})$.

Радоновские триплеты $\beta \equiv\left(\mu, \mu_{1}, \mu_{2}\right)$ и $\gamma \equiv\left(\nu, \nu_{1}, \nu_{2}\right)$ называются эквивалентными $(\beta \sim \gamma)$, если $\mu\left|\mathscr{B}_{c}(T, \mathscr{G})=\nu\right| \mathscr{B}_{c}(T, \mathscr{G})$. Класс эквивалентности в $\mathfrak{R}(T, \mathscr{G})$ радоновского триплета $\beta$ обозначим через $\bar{\beta} \equiv \theta \beta$. Множество всех классов $\bar{\beta}$ для всех радоновских триплетов $\beta \in \mathfrak{R}(T, \mathscr{G})$ обозначим через $\mathfrak{R B}(T, \mathscr{G})$.

Любой элемент $\mathfrak{m} \equiv \bar{\beta}$ из $\mathfrak{R \mathfrak { B }}(T, \mathscr{G})$ называется радоновской бимерой. Если $\beta \in \mathfrak{R}_{b}(T, \mathscr{G})$, то $\mathfrak{m} \equiv \bar{\beta}$ называется ограниченной радоновской бимерой. Семейство всех ограниченных радоновских бимер обозначим через $\mathfrak{R B}_{b}(T, \mathscr{G})$.

В [51; п. II.2] определены операции сложения бимер и умножения их на действительные числа и бинарное отношение порядка $\leqslant$, доказана корректность этих определений. Там же доказано, что семейства $\mathfrak{R} \mathfrak{B}(T, \mathscr{G})$ и $\mathfrak{R} \mathfrak{B}_{b}(T, \mathscr{G})$ являются решеточными линейными пространствами.

В [47; §2, п. 2] была установлена следующая связь между положительными радоновскими мерами и радоновскими бимерами. Для каждой меры $\mu \in \mathfrak{R M}(T, \mathscr{G})_{0}$ возьмем узкую положительную радоновскую меру $\mu_{f} \equiv \mu \mid \mathscr{B}_{c}$, радоновский триплет $\beta \equiv\left(\mu_{f}, \mu, 0\right)$ и положительную радоновскую бимеру $\mathfrak{m} \equiv \bar{\beta}$. Эта процедура определяет отображение $E^{0}: \mathfrak{R M}(T, \mathscr{G})_{0} \rightarrow \mathfrak{R B}(T, \mathscr{G})_{+}$ такое, что $E^{0} \mu \equiv \mathfrak{m}$.

ТЕОРема 14. Пусть $(T, \mathscr{G})$ - хаусдорфово пространство. Тогда отображение $E^{0}: \mathfrak{R M}(T, \mathscr{G})_{0} \rightarrow \mathfrak{R B}(T, \mathscr{G})_{+}$является конусно-линейной биекиией, сохраняющей решеточные операции. При этом для каждой бимеры $\mathfrak{m} \in \mathfrak{R} \mathfrak{B}(T, \mathscr{G})$ существуют единственные мерь $\mu^{\prime}, \mu^{\prime \prime} \in \mathfrak{R M}(T, \mathscr{G})_{0}$ такие, что $\mathfrak{m}=E^{0} \mu^{\prime}-$ $E^{0} \mu^{\prime \prime}, E^{0} \mu^{\prime}=\mathfrak{m}_{+} u E^{0} \mu^{\prime \prime}=-\mathfrak{m}_{-}$.

В [58] было построено продолжение вложения $E^{0}$ на множество всех радоновских мер. Пусть $\mu$ - радоновская мера. Согласно [58; лемма 1.3], ее вариации $v_{+}(\mu)$ и $v^{-}(\mu)$ являются радоновскими мерами, причем хотя бы одна из них конечна. Рассмотрим радоновский триплет $\beta \equiv\left(\mu \mid \mathscr{B}_{c}, v_{+}(\mu),-v^{-}(\mu)\right)$. В [58; п. 5.2] показано, что отображение $E: \mu \mapsto \theta \beta$ семейства $\mathfrak{R M}(T, \mathscr{G})$ всех радоновских мер в пространство $\mathfrak{R B}(T, \mathscr{G})$ всех радоновских бимер определено корректно.

Следуя работе [51; п. II.3], введем интегрирование относительно радоновской бимеры.

Для каждой радоновской меры $\mu$ рассмотрим семейство $B I(T, \mathscr{G}, \mu) \equiv$ $M I(T, \mathscr{B}(T, \mathscr{G}), \mu)$ всех борелевских $\mu$-интегрируемых функиий. Для каждой радоновской бимеры $\mathfrak{m}$ рассмотрим меры $\mu^{\prime}$ и $\mu^{\prime \prime}$ из теоремы 14 и семейство $B I(T, \mathscr{G}, \mathfrak{m}) \equiv B I\left(T, \mathscr{G}, \mu^{\prime}\right) \cap B I\left(T, \mathscr{G}, \mu^{\prime \prime}\right) \quad$ всех борелевских функиий, интегрируемых (по Лебегу) относительно бимеры $\mathfrak{m}$.

Число $i_{\mathfrak{m}}(f) \equiv \int f d \mathfrak{m} \equiv \int f d \mu^{\prime}-\int f d \mu^{\prime \prime}$ называется интегралом (Лебега) от функиии $f \in B I(T, \mathscr{G}, \mathfrak{m})$ относителъно бимеры $\mathfrak{m}$.

Справедлива следующая теорема, связывающая семейства радоновских мер и бимер. 
Теорема 15. Пусть $(T, \mathscr{G})$ - хаусдорфово пространство. Тогда

1) $E \mu=E^{0}\left(v_{+}(\mu)\right)-E^{0}\left(-v^{-}(\mu)\right)$ для всякой $\mu \in \mathfrak{R M}(T, \mathscr{G})$;

2) отображение $E$ инъективно и сохраняет все линейные и решеточные структуры;

3) отображение $E_{b} \equiv E \mid \mathfrak{R M}_{b}(T, \mathscr{G}): \mathfrak{R M}_{b}(T, \mathscr{G}) \rightarrow \mathfrak{R B}_{b}(T, \mathscr{G})$ биективно и является изоморфизмом данных решеточных линейных пространств;

4) для каждой меры $\mu \in \mathfrak{R M}(T, \mathscr{G})$ и любой функиии $f \in B I(T, \mathscr{G}, \mu)$ справедливо равенство $\int f d \mu=\int f d E \mu$.

Таким образом, найдено инъективное вложение семейства $\mathfrak{R M}(T, \mathscr{G})$ всех радоновских мер в решеточное линейное пространство $\mathfrak{R} \mathfrak{B}(T, \mathscr{G})$ всех радоновских бимер, сохраняющее все линейные и решеточные структуры и интеграл Лебега. Это означает, что пространство $\mathfrak{R B}(T, \mathscr{G})$ является естественной решеточной линейной оболочкой семейства $\mathfrak{R M}(T, \mathscr{G})$.

Наличие "бимерной” оболочки позволяет доказать теорему об интегральном представлении $\sigma$-точных функционалов в полном объеме (ср. с теоремой 11$)$. Поэтому ее можно назвать общей параметрической теоремой о характеризаиии интегралов по радоновским бимерам. Она появилась в работах [25], [57] с кратким доказательством, а в [58] - с полным.

Теорема 16. Пусть $(T, \mathscr{G})$ - хаусдорфово пространство и $A(T)$-усекаемое решеточное линейное подпространство в $S(T, \mathscr{G})$, обладающее свойством $\left(E_{\sigma}\right)$ или свойством $(E) \&(D)$. Тогда

1) $I(A(T), \mathfrak{R B}(T, \mathscr{G}))=A(T)^{\Delta}$ для $A(T) \subset \bigcap(B I(T, \mathscr{G}, \mathfrak{m}) \mid \mathfrak{m} \in \mathfrak{R B}(T, \mathscr{G}))$;

2) $\left.I\left(A(T), \mathfrak{R B} \mathfrak{B}_{b}(T, \mathscr{G})\right)=A(T)^{(}\right)$.

Более того, отображение $M: \mathfrak{m} \mapsto i_{\mathfrak{m}} \mid A(T)$ является изоморбизмом соответствующих решеточных линейных пространств.

Наличие естественной линейной оболочки для семейства радоновских мер $\mathfrak{R M}(T, \mathscr{G})$ и общей теоремы 16 позволяет завершить упомянутое выше восстановление радоновской меры $\mu$ по функционалу $\varphi \in\left(A(T)^{\triangle}\right)_{(?)}$.

Функционал $\varphi: A(T) \rightarrow \mathbb{R}$ назовем натуралъным, если

$$
\bar{b}(\varphi) \equiv \sup \left\{\varphi f \mid f \in A(T)_{+} \text {и } f \leqslant \mathbf{1}\right\}<\infty
$$

и

$$
\underline{b}(\varphi) \equiv \inf \left\{\varphi f \mid f \in A(T)_{+} \text {и } f \leqslant \mathbf{1}\right\}>-\infty,
$$

т. е. хотя бы одна из указанных границ конечна. Множество всех натуральных $\sigma$-точных линейных функционалов обозначим через $\left(A(T)^{\Delta}\right)_{\text {nat }}$.

Свойство натуральности выступает в роли уточняющего свойства (?), фигурировавшего выше. Действительно, если функционал $\varphi$ - натуральный, то хотя бы одна из восстанавливаемых положительных радоновских мер $\mu_{1}(\varphi)$ и $\mu_{2}(\varphi)$ является конечной. Поэтому вместо абстрактной бимеры $\mathfrak{m} \equiv \theta\left(\mu_{1}(\varphi), \mu_{2}(\varphi)\right)$ можно взять радоновскую меру $\mu \equiv \mu_{1}(\varphi)-\mu_{2}(\varphi)$. С помощью теорем 11,15 и 16 мы приходим к следующей общей параметрической теореме о характеризации интегралов по всем радоновским мерам [58]-[60]. 
Теорема 17. Пусть $(T, \mathscr{G})$ - хаусдорфово пространство и $A(T)$ - усекаемое решеточное линейное подпространство в пространстве $S(T, \mathscr{G}) \cap U I(T$, $\mathscr{G}, \mathfrak{R M}(T, \mathscr{G}))$, обладающее свойством $\left(E_{\sigma}\right)$ или свойством $(E) \&(D)$. Тогда $I(A(T), \mathfrak{R M}(T, \mathscr{G}))=\left(A(T)^{\triangle}\right)_{\text {nat }}$.

Более того, биективное отображение $\mu \mapsto i_{\mu} \mid A(T)$ из $\mathfrak{R M}(T, \mathscr{G})$ в $\left(A(T)^{\Delta}\right)_{\text {nat }}$ сохраняет все линейные и решеточные структуры, наследуемые семейством мер $\mathfrak{R M}(T, \mathscr{G})$ и семейством функиионалов $\left(A(T)^{\Delta}\right)_{\text {nat }}$ из их решеточных линейных оболочек.

СлЕДСТВИЕ 1. Если $(T, \mathscr{G})$ - хаусдорфово пространство, то

$$
I\left(S_{c}(T, \mathscr{G}), \mathfrak{R M}(T, \mathscr{G})\right)=\left(S_{c}(T, \mathscr{G})^{\Delta}\right)_{\text {nat }} .
$$

СлЕДСТвиЕ 2. Если $(T, \mathscr{G})$ - хаусдорфово пространство, то

$$
I\left(S(T, \mathscr{G}), \mathfrak{R M} \mathfrak{M}_{b}(T, \mathscr{G})\right)=S(T, \mathscr{G})^{\Delta}
$$

и отображсение $\mu \mapsto i_{\mu} \mid S(T, \mathscr{G})$ является изоморбизмом решеточных линейных пространств.

СлЕДСТвиЕ 3 (теорема Бурбаки). Если $(T, \mathscr{G})$ - тихоновское пространство, mo

$$
I\left(C_{b}(T, \mathscr{G}), \mathfrak{R M} \mathfrak{M}_{b}(T, \mathscr{G})\right)=C_{b}(T, \mathscr{G})^{\pi}
$$

и отображение $\mu \mapsto i_{\mu} \mid C_{b}(T, \mathscr{G})$ также является изоморфизмом.

СлЕДСТВИЕ 4. Если $(T, \mathscr{G})$ - локально компактное пространство, то

$$
\begin{aligned}
I\left(C_{c}(T, \mathscr{G}), \mathfrak{R M}(T, \mathscr{G})\right) & =\left(C_{c}(T, \mathscr{G})^{\sim}\right)_{\text {nat }}, \\
I\left(C_{c}(T, \mathscr{G}), \mathfrak{R M}(T, \mathscr{G})_{0}\right) & =\left(C_{c}(T, \mathscr{G})^{\sim}\right)_{+} .
\end{aligned}
$$

СледствиЕ 5 (теорема Радона-Банаха-Сакса-Какутани). Eсли (T, $\mathscr{G})$ компактное пространство, то

$$
I(C(T, \mathscr{G}), \mathfrak{R M}(T, \mathscr{G}))=C(T, \mathscr{G})^{\sim}
$$

и отображение $\mu \mapsto i_{\mu} \mid C(T, \mathscr{G})$ также является изоморфизмом.

Заключительная теорема 17 обобщает теорему 11 и поэтому влечет как простые частные случаи теоремы 2-5 и 10. Однако теорема 17 не отменяет теорему 11, поскольку базируется на ней.

\section{Список литературы}

[1] J. Hadamard, "Sur les opérations fonctionnelles", C.R. Acad. Sci. Paris, 136 (1903), 351-354.

[2] M. Fréchet, "Sur les opérations linéaires", Trans. Amer. Math. Soc., 5:4 (1904), 493-499.

[3] Н. Бурбаки, Интегрирование: Меры. Интегрирование мер, Наука, М., 1967, 396 с.; пер. с франц.: N. Bourbaki, Élements de mathématique, Actualités Sci. Ind., 1244, Hermann, Paris, 1952, 237 pp. 
[4] F. Riesz, "Sur les opérations fonctionnelles linéaires", C. R. Acad. Sci. Paris, 149 (1910), 974-977.

[5] И.П. Натансон, Теория функиий вещественной переменной, ГИТТЛ, М.-Л., 1950, 399 с.; 9-е изд.: Лань, СПб., 2007, 727 с.; англ. пер.: I. P. Natanson, Theory of functions of a real variable, Frederick Ungar Publishing Co., New York, 1955, 277 pp.

[6] J. Radon, "Theorie und Anwendungen der absolut additiven Mengenfunktionen", Wien. Ber., 122 (1913), 1295-1438.

[7] M. Fréchet, "Sur l'intégrale d'une fonctionnelle étendue à un ensemble abstrait", Bull. Soc. Math. France, 43 (1915), 248-265.

[8] Н. Бурбаки, Интегрирование: Меры на компактных пространствах. Продолжение меры. Интегрирование мер. Меры на отделимых пространствах, Наука, M., 1977, 600 с.; пер. с франц: N. Bourbaki, Éléments de mathématique. v. XIII: Livre VI: Intégration. Chapitres I-IV: Inégalités de convexité, Espaces de Riesz, Mesures sur les espaces localement compacts, Prolongement d'une mesure, Espaces $L^{p}$, Deuxième édition revue et augmentée. Actualités Scientifiques et Industrielles, 1175, Hermann, Paris, 1965, 283 pp.; v. XXI: Livre VI: Intégration. Chapitre V: Intégration des mesures, Deuxième édition revue et augmentée. Actualités Scientifiques et Industrielles, 1244, 1967, 154 pp.; v.XXXV: Livre VI: Intégration. Chapitre IX: Intégration sur les espaces topologiques séparés, Deuxième édition revue et augmentée. Actualités Scientifiques et Industrielles, 1343, 1969, 133 pp.

[9] Ф. Хаусдорф, Теория множеств, УРСС, М., 2004, 303 с.; пер. с нем.: F. Hausdorff, Grundzüge der Mengenlehr, Viet, Leipzig, 1914, ISBN: 5-354-00842-5, 476 pp.

[10] Z. Semadeni, Banach spaces of continuous functions. v. I, Monografie Matematyczne, 55, PWN, Warsaw, 1971, 584 pp.

[11] В.И. Богачёв, Основь теории мерь, Учебное пособие. т. І, РХД, М.-Ижевск, 2006, ISBN: 5-93972-432-9, 583 с.; т. 2, ISBN: 5-93972-433-7, 679 с.; англ. пер.: V.I. Bogachev, Measure theory. v. I, Springer-Verlag, Berlin, 2007, ISBN: 978-3-54034513-8, 500 pp.; v. II, ISBN: 3-540-34513-2, 575 pp.

[12] S. Saks, Theory of the integral, G. E. Stechert, New York, 1937, 347 pp.; рyc. пер.: C. Сакс, Теория интеграла, Факториал, М., 2004.

[13] S. Saks, "Integration in abstract metric spaces", Duke Math. J., 4:2 (1938), 408-411.

[14] Н. Данфорд, Дж. Т. Шварц, Линейные операторы. Общая теория, ИЛ, М., 1962, 895 с.; пер. с англ.: N. Dunford, J. T. Schwartz, Linear Operators. I. General Theory, Pure Appl. Math., 7, Interscience Publishers, New York-London, 1958, 858 pp.

[15] S. Kakutani, "Concrete representation of abstract (M)-spaces", Ann. of Math. (2), 42:4 (1941), 994-1024.

[16] K. Jacobs, Measure and integral, Probability and Mathematical Statistics, Academic Press, New York-London, 1978, ISBN: 0-12-378550-2, 575 pp.

[17] E. Hewitt, K. Stromberg, Real and abstract analysis. A modern treatment of the theory of functions of a real variable, Springer-Verlag, New York, 1965, 476 pp.

[18] D. H. Fremlin, Topological Riesz spaces and measure theory, Cambridge Univ. Press, London-New York, 1974, 266 pp.

[19] D. H. Fremlin, Measure theory. v. 1: The irreducible minimum, Torres Fremlin, Colchester, 2004, ISBN: 0-9538129-0-1, 108 pp.; v. 2: Broad foundations, 2003, ISBN: 0-9538129-2-8, 563 pp.; v. 3: Measure algebras, 2004, ISBN: 0-9538129-3-6, 693 pp.; v. 4: Topological measure spaces. Part I, II, 2006, ISBN: 0-9538129-4-4, 528+439 pp.; v. 5: Set-theoretic measure theory. Part I, II, 2008, ISBN: 978-0-9538129-5-0/pt. I, 978-0-9538129-6-7/pt. II, 292 pp.

[20] Б. З. Вулих, Введение в теорию полуупорядоченных пространств, Физматгиз, M., 1961, 407 с.; англ. пер.: B.Z. Vulikh, Introduction to the theory of partially ordered spaces, Wolters-Noordhoff Scientific Publications, Groningen, 1967, 387 pp. 
[21] П. Халмош, Теория меры, ИЛ, М., 1953, 291 с.; пер. с англ.: P. R. Halmos, Measure theory, Van Nostrand, New York, 1950, 304 pp.

[22] E. Hewitt, "Integration on locally compact spaces. I", Univ. Washington Publ. Math., 3 (1952), 71-75.

[23] R. E. Edwards, "A theory of Radon measures on locally compact spaces", Acta Math., 89:1 (1953), 133-164.

[24] Ю.В. Прохоров, "Сходимость случайных процессов и предельные теоремы теории вероятностей", Теория вероятн. и ее примен., 1:2 (1956), 177-238.

[25] В. К. Захаров, "Проблема характеризации радоновских интегралов", Докл. РАН, 385:6 (2002), 735-737; англ. пер.: V. K. Zakharov, "Problem of characterization of Radon integrals", Dokl. Math., 66:1 (2002), 118-120.

[26] F. Topsoe, Topology and measure, Lecture Notes in Math., 133, Springer-Verlag, Berlin-New York, 1970, 79 pp.

[27] N. Dinculeanu, Vector measures, Internat. Ser. Monogr. Pure and Appl. Math., 95, Pergamon Press, Oxford - New York - Toronto, ON; VEB Deutscher Verlag der Wissenschaften, Berlin, 1967, 432 pp.

[28] A.D. Alexandroff, "Additive set functions on abstract spaces", Матем. сб., 8:2 (1940), 307-348; 9:3 (1941), 563-628; 13:2-3 (1943), 169-238.

[29] M. N. Stone, "Notes on integration. I", Proc. Natl. Acad. Sci. USA, 34:7 (1948), 336-342; "Notes on integration. II", 34:9 (1948), 447-455; "Notes on integration. III", 34:10 (1948), 483-490; "Notes on integration. IV", 35:1 (1949), 50-58.

[30] D. Pollard, F. Topsoe, "A unified approach to Riesz type representation theorems", Studia Math., 54:2 (1975), 173-190.

[31] F. Topsoe, "Further results on integral representations", Studia Math., 55:3 (1976), 239-245.

[32] F. Topsoe, "Radon measures, some basic constructions", Measure theory and its applications (Sherbrooke, QC, 1982), Lecture Notes in Math., 1033, Springer, Berlin, 1983, 303-311.

[33] B. Anger, C. Portenier, Radon integrals. An abstract approach to integration and Riesz representation through function cones, Progr. in Math., 103, Birkhäuser, Boston, MA, 1992, ISBN: 0-8176-3630-7, 332 pp.

[34] H. König, "The Danielle-Stone-Riesz representation theorem", Operator theory in function spaces and Banach lattices, Oper. Theory Adv. Appl., 75, Birkhäuser, Basel, 1995, 191-222.

[35] H. König, Measure and integration. An advanced course in basic procedures and applications, Springer-Verlag, Berlin, 1997, ISBN: 3-540-61858-9, 260 pp.

[36] H. König, "On the inner Daniell-Stone and Riesz representation theorems", Doc. Math., 5 (2000), 301-315.

[37] H. König, "Measure and integration: an attempt at unified systematization", Rend. Istit. Mat. Univ. Trieste, 34:1-2 (2002), 155-214; http://www.math.uni-sb.de/ service/preprints/preprint42.pdf.

[38] H. König, "New version of the Danielle-Stone-Riesz representation theorem", Positivity, 12:1 (2008), 105-118; phttp://www.math.uni-sb.de/service/preprints/ reprint179.pdf.

[39] V.K. Zakharov, "Alexandrovian cover and Sierpińskian extension", Studia Sci. Math. Hungar., 24:2-3 (1989), 93-117.

[40] В.К. Захаров, "Связь между полным кольцом частных кольца непрерывных функций, регулярным пополнением и расширениями Хаусдорфа-Серпинского", УМH, 45:6 (1990), 133-134; англ. пер.: V. K. Zakharov, "The connection between the complete ring of quotients of the ring of continuous functions, regular completion, and Hausdorff-Sierpiński extensions", Russian Math. Surveys, 46:6 (1990), 177-178. 
[41] F. Hausdorff, "Über halbstetige Functionen und deren Verallgemeinerung", Math. Z., 4 (1919), 292-309.

[42] W. Sierpiński, "Sur les fonctions développables en séries absolument convergentes de fonctions continues", Fund. Math., 2 (1921), 15-27.

[43] В.К. Захаров, "Связи между расширением Лебега и расширением Бореля первого класса и между соответствующими им прообразами", Изв. АН СССР. Сер. матем., 54:5 (1990), 928-956; англ. пер.: V. K. Zakharov, "Connections between the Lebesgue extension and the Borel extension of the first class, and between the preimages corresponding to them", Math. USSR-Izv., 37:2 (1991), 273-302.

[44] В. К. Захаров, Т. В. Родионов, "Классификация борелевских множеств и функций на произвольном пространстве", Матем. сб., 199:6 (2008), 49-84; англ. пер.: V.K. Zakharov, T. V. Rodionov, "Classification of Borel sets and functions for an arbitrary space", Sb. Math., 199:6 (2008), 833-869.

[45] В.К. Захаров, "Прообраз Гордона пространства Александрова как окружаемое накрытие", Изв. РАН. Сер. матем., 56:2 (1992), 427-448; англ. пер.: V.K. Zakharov, "The Gordon preimage of an Aleksandrov space as an enclosed covering", Russian Acad. Sci. Izv. Math., 40:2 (1993), 405-424.

[46] В.К. Захаров, "Расширение Аренса кольца непрерывных функций”, Алгебра и анализ, 4:2 (1992), 135-153; англ. пер.: V. K. Zakharov, "Arens extension of a ring of continuous functions", St. Petersburg Math. J., 4:1 (1993), 131-148.

[47] В. К. Захаров, А. В. Михалёв, "Проблема общего радоновского представления для произвольного хаусдорфова пространства", Изв. РАН. Сер. матем., 63:5 (1999), 37-82; англ. пер.: V. K. Zakharov, A. V. Mikhalev, "The problem of general Radon representation for an arbitrary Hausdorff space", Izv. Math., 63:5 (1999), 881-921.

[48] В.К. Захаров, "Новые классы функций, связанные с общими семействами множеств", Докл. РАН, 407:2 (2006), 167-171; англ. пер.: V. K. Zakharov, "New classes of functions related to general families of sets", 73:2 (2006), 197-201.

[49] В. К. Захаров, Т. В. Родионов, "Класс равномерных функций и его соотношение с классом измеримых функций", Матем. заметки, 84:6 (2008), 809-824; англ. пер.: V.K. Zakharov, T. V. Rodionov, "A class of uniform functions and its relationship with the class of measurable functions", Math. Notes, 84:5-6 (1999), 756-770.

[50] В.К. Захаров, "Расширения кольца непрерывных функций, порожденные регулярным, счетно-делимым и полным кольцами частных, и соответствующие им прообразы", Изв. РАН. Сер. матем., 59:4 (1995), 15-60; англ. пер.: V.K. Zakharov, "Extensions of the ring of continuous functions generated by regular, countably-divisible, complete rings of quotients, and their corresponding pre-images", Izv. Math., 59:4 (1995), 667-720.

[51] В. К. Захаров, А. В. Михалёв, "Интегральное представление для радоновских мер на произвольном хаусдорфовом пространстве", Фундамент. и прикл. матем., 3:4 (1997), 1135-1172.

[52] В. К. Захаров, А. В. Михалёв, "Проблема Радона для регулярных мер на произвольном хаусдорфовом пространстве", Фундамент. и прикл. матем., 3:3 (1997), 801-808.

[53] В. К. Захаров, А. В. Михалёв, "Проблема интегрального представления для радоновских мер на произвольном хаусдорфовом пространстве”, Докл. РАН, 360:1 (1998), 13-15; англ. пер.: V. K. Zakharov, A. V. Mikhalev, "The problem of integral representation for Radon measures on a Hausdorff space", Dokl. Math., 57:3 (1998), 337-339.

[54] V. K. Zakharov, A. V. Mikhalev, "Riesz-Radon problem of characterisation of Radon integrals", Kolmogorov and contemporary mathematics, Dekker, New York, 2003, 260-261. 
[55] В. К. Захаров, А. В. Михалёв, "Связь между интегральными радоновскими представлениями для локально компактного и хаусдорфова пространств", Фундамент. и прикл. матем., 7:1 (2001), 33-46.

[56] В.К. Захаров, А.В. Михалёв, "Проблема общего радоновского представления для произвольного хаусдорфова пространства. II", Изв. РАН. Сер. матем., 66:6 (2002), 3-18; англ. пер.: V. K. Zakharov, A. V. Mikhalev, "The problem of general Radon representation for an arbitrary Hausdorff space. II", Izv. Math., 66:6 (2002), 1087-1101.

[57] В.К. Захаров, "Проблема Рисса-Радона характеризации интегралов и слабая компактность радоновских мер", Тр. МИАН, 248 (2005), 106-116; англ. пер.: V.K. Zakharov, "The Riesz-Radon problem of the characterization of integrals, and the weak compactness of Radon measures", Proc. Steklov Inst. Math., 2005:1 (2005), $101-110$.

[58] В. К. Захаров, А. В. Михалёв, Т. В. Родионов, "Описание радоновских интегралов как линейных функционалов", Фундамент. и прикл. матем. (в печати).

[59] В. К. Захаров, А. В. Михалёв, Т. В. Родионов, "Проблема Рисса-Радона-Фреше характеризации радоновских интегралов: ограниченные меры; положительные меры; бимеры; общие радоновские меры", Современные проблемы математики, механики и их приложений, Материалы междунар. конф., посв. 70-летию ректора МГУ акад. В. А. Садовничего, Университетская книга, М., 2009, 91-92.

[60] В. К. Захаров, А. В. Михалёв, Т. В. Родионов, "Проблема характеризации общих радоновских интегралов", Докл. РАН, 433:6 (2010).

В. K. Захаров (V. K. Zakharov)

Московский государственный университет им. М. В. Ломоносова;

Центр новых информационных технологий МГУ им. М. В. Ломоносова

E-mail: zakharov_valeriy@list.ru

\section{А. В. Михалёв (А. V. Mikhalev)}

Центр новых информационных технологий МГУ им. М. В. Ломоносова

E-mail: mikhalev@rector.msu.ru

\section{T. В. Родионов (T. V. Rodionov)}

Московский государственный университет им. М. В. Ломоносова

E-mail: ttvvrr@yandex.ru
Поступила в редакцию 25.02 .2010 